\title{
INTRAGROUP AND GALAXY-LINKED DIFFUSE X-RAY EMISSION IN HICKSON COMPACT GROUPS
}

\author{
Tyler D. Desjardins ${ }^{1}$, Sarah C. Gallagher ${ }^{1}$, Panayiotis Tzanavaris ${ }^{2}$, John S. Mulchaey ${ }^{3}$, William N. Brandi ${ }^{4,7}$, \\ Jane C. Charlton ${ }^{4}$, Gordon P. Garmire ${ }^{4}$, Caryl Gronwall ${ }^{4,7}$, Ann E. Hornschemeier ${ }^{2}$, Kelsey E. Johnson ${ }^{5}$, \\ IRAKLIS S. KonSTANTOPOULOS ${ }^{4}$, AND ANN I. ZaBLUdOFF ${ }^{6}$ \\ ${ }^{1}$ Department of Physics and Astronomy, The University of Western Ontario, London, ON N6A 3K7, Canada; tdesjar@uwo.ca \\ ${ }^{2}$ Laboratory for X-ray Astrophysics, NASA/Goddard Space Flight Center, Greenbelt, MD 20771, USA \\ ${ }^{3}$ Carnegie Observatories, 813 Santa Barbara Street, Pasadena, CA 91101, USA \\ ${ }^{4}$ Department of Astronomy and Astrophysics, 525 Davey Laboratory, The Pennsylvania State University, University Park, PA 16802, USA \\ ${ }^{5}$ Department of Astronomy, University of Virginia, P.O. Box 3813, Charlottesville, VA 22904, USA \\ ${ }^{6}$ Steward Observatory, University of Arizona, 933 North Cherry Avenue, Tucson, AZ 95721, USA \\ Received 2012 May 7; accepted 2012 December 3; published 2013 January 16
}

\begin{abstract}
Isolated compact groups (CGs) of galaxies present a range of dynamical states, group velocity dispersions, and galaxy morphologies with which to study galaxy evolution, particularly the properties of gas both within the galaxies and in the intragroup medium. As part of a large, multiwavelength examination of CGs, we present an archival study of diffuse X-ray emission in a subset of nine Hickson compact groups (HCGs) observed with the Chandra $X$-Ray Observatory. We find that seven of the groups in our sample exhibit detectable diffuse emission. However, unlike large-scale emission in galaxy clusters, the diffuse features in the majority of the detected groups are linked to the individual galaxies, in the form of both plumes and halos likely as a result of vigourous star formation or activity in the galaxy nucleus, as well as in emission from tidal features. Unlike previous studies from earlier X-ray missions, HCGs 31, 42, 59, and 92 are found to be consistent with the $L_{X}-T$ relationship from clusters within the errors, while HCGs 16 and 31 are consistent with the cluster $L_{X}-\sigma$ relation, though this is likely coincidental given that the hot gas in these two systems is largely due to star formation. We find that $L_{X}$ increases with decreasing group $\mathrm{H}$ I to dynamical-mass ratio with tentative evidence for a dependence in X-ray luminosity on $\mathrm{H}$ i morphology whereby systems with intragroup $\mathrm{H}$ I indicative of strong interactions are considerably more X-ray luminous than passively evolving groups. We also find a gap in the $L_{X}$ of groups as a function of the total group specific star formation rate. Our findings suggest that the hot gas in these groups is not in hydrostatic equilibrium and these systems are not low-mass analogs of rich groups or clusters, with the possible exception of HCG 62.
\end{abstract}

Key words: galaxies: evolution - galaxies: groups: general - X-rays: galaxies

Online-only material: color figures

\section{INTRODUCTION}

As the majority of galaxies in the nearby universe are found within gravitationally bound groups (e.g., Tully 1987; Small et al. 1999; Karachentsev 2005), understanding the physical processes at work in these systems is fundamental to galaxy formation, evolution, and cosmological theory. Though a significant fraction may be condensed structures embedded within largely extended, loosely bound systems (Tovmassian et al. 2006; Mendel et al. 2011), Hickson compact groups (HCGs; Hickson 1982) in particular are useful tools for studying tidally enhanced galaxy evolution in the nearby universe because they are isolated from other nearby galaxies due to their selection criteria, have low-velocity dispersions (usually $\lesssim 200 \mathrm{~km} \mathrm{~s}^{-1}$ ), and exhibit high number densities (typically 3-4 galaxies of comparable luminosity within several galaxy radii of one another). These properties combined encourage numerous gravitational interactions between group members.

Because the crossing time of compact groups (CGs) is short $\left(\lesssim 0.02 t_{H_{0}}\right.$; Diaferio et al. 1994), simulations indicate that group members should have entirely merged into single massive galaxies on timescales of $\sim 1$ Gyr. Hypotheses concerning the continued existence of CGs have included that these systems are either recently formed (e.g., Mendes de Oliveira et al.

\footnotetext{
7 Also at Institute for Gravitation and the Cosmos, The Pennsylvania State University, University Park, PA 16802, USA.
}

2005), that they are chance alignments within larger structures (e.g., Mamon 1986), or that CGs are perpetually formed within collapsing rich groups (e.g., Diaferio et al. 1994). However, all three proposed scenarios have evidence that contradicts them, such as the wide range in ages of star clusters in CGs (Konstantopoulos et al. 2010, 2012), the low surface brightness tidal debris and disturbed disk morphologies present in many groups (e.g., HCGs 16, 31, and 92; Mendes de Oliveira et al. 1998; Gallagher et al. 2010; Fedotov et al. 2011), and indications that a small fraction of CG members are the result of mergers (Mendes de Oliveira et al. 2005). Clearly these systems are much more complicated than first suspected, and understanding the origin and physical processes responsible for maintaining CGs over timescales in excess of several Gyr is important for refining cosmological simulations.

Verdes-Montenegro et al. (2001) presented an evolutionary scenario in which the spiral-rich CGs with most of their neutral $\mathrm{H}$ I gas confined to the galaxy disks experience successive tidal encounters which liberate the cool gas from the galaxies into the intragroup medium (IGM) in the form of tails and bridges. This progresses to one of two evolutionary end points: (1) a group in which the liberated $\mathrm{HI}$ is shock-heated to X-ray temperatures or (2) a group with a large, low-velocity dispersion H I halo. In a study of five groups, Freeland et al. (2009) found that the amount of $\mathrm{H}$ I tidally removed from galaxy disks is related to the compactness of the group. Therefore, CGs should be very efficient at dispersing their neutral gas 
into the intragroup medium. If enough gas mass is removed from the disks and heated sufficiently, the group may be considered a fossil group. Jones et al. (2003) defined fossil groups as those with $L_{X} \geqslant 10^{42} h_{50}^{-2} \mathrm{erg} \mathrm{s}^{-1}$ and a difference in $R$-band magnitude of $\Delta m \geqslant 2.0$ mag between the two brightest group members. The possible evolution of HCGs into fossil groups represents the most similar to clusters these systems may become. We note that tidal stripping of gas from galaxy disks is not necessarily the only source of baryons for the intragroup medium. Simulations of galaxy clusters at high redshift have shown that cold mode accretion of gas from the surrounding intergalactic medium can be a substantial source of baryons (e.g., Kereš et al. 2005); however, it is unclear how significant this accretion may be for low-redshift groups.

Observations of diffuse X-ray emission are particularly helpful in placing constraints on the properties of CGs because the increased likelihood of tidal encounters implies that a significant fraction of the gas mass in these systems may be in the form of a hot plasma. Previous ROSAT (e.g., Ponman et al. 1996; Mulchaey \& Zabludoff 1998; Helsdon et al. 2001; Mulchaey et al. 2003) and Chandra (e.g., Rasmussen et al. 2008; Rasmussen \& Ponman 2009; Sun et al. 2009) analyses of diffuse X-ray emission in galaxy groups have specifically treated groups of galaxies as low-mass analogs of galaxy clusters. Early studies found that, within large errors, galaxy groups were consistent with the established relationships between bolometric X-ray luminosity $L_{X}$ and gas temperature $\left(L_{X}-T\right)$ as well as $L_{X}$ and velocity dispersion $\left(L_{X}-\sigma\right)$ found from observations of large samples of galaxy clusters. With improved instrumentation and more statistically robust data sets, it was shown that CGs deviate from the established $L_{X}-T$ cluster relation (groups are fainter for a given temperature); however, the $L_{X}-\sigma$ relation was still found to be consistent, though with a large scatter (cf. Figure 12 in Ponman et al. 1996).

Having performed the first extensive X-ray survey of CGs using data from both the ROSAT All-Sky Survey (RASS) and pointed ROSAT PSPC observations, Ponman et al. (1996) found that 22 out of 85 observed HCGs had diffuse X-ray emission above their detection limits. From the data, the authors statistically estimated that $\sim 75 \%$ of HCGs have a diffuse $\mathrm{X}$-ray luminosity above $1.3 \times 10^{41} \mathrm{erg} \mathrm{s}^{-1}$. While diffuse $\mathrm{X}$-ray emission was previously thought to be limited to groups containing only E/S0 members, Ponman et al. (1996) found that groups containing spiral galaxies exhibit diffuse $\mathrm{X}$-ray emission as well, however, the X-ray luminosity functions of spiraldominated groups show that they tend to be fainter than E/SOdominated groups to a high statistical significance. Additionally, a weak anti-correlation was found between $L_{X}$ and spiral galaxy fraction.

Studies of CGs such as HCG 31, which shows multiple interactions among its low-mass members, have shown evidence for hierarchical structure formation characteristic of environments at higher redshifts $(z \sim 1-2$; Gallagher et al. 2010). Additionally, a gap in both the mid-IR colors and specific star formation rates (SSFRs; i.e., SFR normalized by stellar mass) of HCGs compared to galaxies in other environments suggests that the galaxies in these systems undergo accelerated evolution from star-forming "blue-cloud" galaxies to the quiescent "red sequence" (Johnson et al. 2007; Walker et al. 2010, 2012; Tzanavaris et al. 2010). This rapid evolution is supported by the H I deficiency observed in most HCG members relative to spiral galaxies in the field (Huchtmeier 1997; Verdes-Montenegro et al. 2001).
The rapid evolution of HCG galaxies from gas-rich and starforming to gas-poor and quiescent raises the following question: how is the neutral gas being processed in HCGs, i.e., is most of the H I mass converted into stellar mass or is it ionized and expelled into the intragroup medium, and in what proportions do these mechanisms operate in individual groups? Previous papers have examined the star cluster populations in several HCGs (e.g., Gallagher et al. 2001; Konstantopoulos et al. 2010; Fedotov et al. 2011) to study the consumption of gas by star formation; however, there has been no investigation of how the HI gas is heated to X-ray temperatures in these systems in the context of group evolution. In this work, as part of a multiwavelength campaign to study the evolution of both the galaxies in CGs and the group environment itself, we present the results of a study of the diffuse X-ray emission from nearby CGs $(z \lesssim 0.015)$ using Chandra observations. Specifically, we inspect the morphology of the hot gas in CGs using improved spatial resolution compared to previous studies that analyzed ROSAT data; we re-examine the $L_{X}$ scaling relations with temperature and velocity dispersion; and we compare the hot gas in groups to the neutral $\mathrm{H}$ I gas to investigate how the $\mathrm{X}$-ray gas is built up in these systems.

First, we describe the Chandra observations and methods for reducing the events files in Section 2. In Section 3, we discuss the extraction of the X-ray spectra, as well as the models we used to fit the data. Our model-derived measurements are presented in Section 4 in addition to a discussion of the results of our study in the larger context of galaxy evolution and the evolution of the group environment itself. We summarize our findings and discuss the future steps of our investigation into the diffuse X-ray properties of HCGs in Section 5. The Appendix gives specific information on the extraction and modeling of the diffuse emission in the detected groups. Throughout this work, we assume a cosmology of $H_{0}=70 \mathrm{~km} \mathrm{~s}^{-1} \mathrm{Mpc}^{-1}$, $\Omega_{\mathrm{M}}=0.27$, and $\Omega_{\Lambda}=0.73$. We also caution the reader that we allude several times to CGs possibly becoming more cluster-like as they evolve. In this context, we refer to the hot gas distribution, not necessarily to the richness of the group; therefore, the term cluster-like is used to describe groups with a large fraction of E/SO galaxies and a shared, hot intragroup medium.

\section{SAMPLE DEFINITION, OBSERVATIONS, AND DATA REDUCTION}

Objects for study were selected on the basis of data available from the archive at the Chandra X-ray Center (CXC) for CGs that are part of a sample designed to study star formation and galaxy evolution in the CG environment (see Johnson et al. 2007 for more information regarding the original sample; HCG 92 was subsequently added based on the availability of highresolution, deep imaging data). To this end, the sample consists of groups at small enough distances $(z \lesssim 0.015$, excepting HCG 92) to allow for high spatial resolution imaging over a wide range of wavelengths. In addition to our new Chandra observations of HCGs 7, 22, 31, and 59 (PIs: Garmire [7, 22] and Gallagher [31, 59]), we selected archival data that covered the entirety of each group, rather than only individual group members, to search for hot, ionized gas in the intragroup medium, bringing the total number in our study of diffuse X-ray emission to nine groups. HCGs 2, 19, 48, and 61, which were included in the Johnson et al. (2007) sample, were omitted from our study due to the lack of suitable Chandra imaging data.

All of the groups were observed at the ACIS-S aimpoint with the exception of HCG 90, which was observed at the ACIS-I 
Table 1

Chandra Observation Information

\begin{tabular}{|c|c|c|c|c|c|c|c|}
\hline $\mathrm{HCG}$ & $\begin{array}{l}\text { Chandra } \\
\text { ObsID }\end{array}$ & Date & $\begin{array}{c}\text { Extraction Area } \\
\left(\operatorname{arcmin}^{2}\right)\end{array}$ & $\begin{array}{l}\text { Total Counts } \\
(0.7-3.0 \mathrm{keV})\end{array}$ & $\begin{array}{l}\text { Exp. } \\
(\mathrm{ks})\end{array}$ & $\begin{array}{l}\text { Bkg Counts } \\
(0.7-3.0 \mathrm{keV})\end{array}$ & $\begin{array}{c}\text { Bkg Exp. }^{\mathrm{c}} \\
(\mathrm{ks})\end{array}$ \\
\hline \multirow[t]{2}{*}{$7^{d}$} & $8171^{\mathrm{d}}$ & 2007 Sep 13 & 48.2 & 2419 & 19.2 & 57670 & 900 \\
\hline & $9588^{d}$ & 2007 Sep 16 & & & 16.7 & & \\
\hline 16 & 923 & 2000 Nov 16 & 26.9 & 1270 & 12.6 & 6209 & 110 \\
\hline $22^{\mathrm{e}}$ & 8172 & 2006 Nov 23 & 44.7 & 2078 & 31.8 & 27973 & 450 \\
\hline $31^{\mathrm{e}}$ & 9405 & 2007 Nov 15 & 8.70 & 636 & 35.6 & 5433 & 450 \\
\hline 42 & 3215 & $2002 \operatorname{Mar} 26$ & 36.5 & 7600 & 31.7 & 15882 & 450 \\
\hline $59^{e}$ & 9406 & 2008 Apr 12 & 24.7 & 1457 & 38.4 & 15373 & 450 \\
\hline \multirow[t]{3}{*}{62} & 921 & 2000 Jan 25 & 7.54 & 31053 & 48.5 & 2604 & 145 \\
\hline & $10462^{d}$ & 2009 Mar 2 & 7.54 & 55302 & 67.1 & 247702 & 900 \\
\hline & $10874^{\mathrm{d}}$ & 2009 Mar 2 & & & 51.4 & & \\
\hline 90 & 905 & $2000 \mathrm{Jul} 2$ & 68.3 & 24821 & 49.5 & 166640 & 450 \\
\hline \multirow[t]{2}{*}{92} & 789 & 2000 Jul 9 & 19.3 & 2545 & 19.7 & 4663 & 110 \\
\hline & 7924 & 2007 Aug 17 & 19.3 & 10656 & 93.2 & 27337 & 450 \\
\hline
\end{tabular}

Notes.

a Total area over which the events were extracted.

b Source + background in science observation.

${ }^{\mathrm{c}}$ Exposure time of the ACIS stowed background files.

${ }^{\mathrm{d}}$ Count information listed for merged spectra.

${ }^{\mathrm{e}}$ Counts evaluated over 0.7-1.7 keV (see Section 3.2).

aimpoint due to its large angular extent (the diffuse emission of HCG 90 falls mostly on the I0 CCD, however, some is present along the edges of the other I array detectors as well). The individual ACIS CCDs have a field of view of $8.3 \times 8.3$, which is comparable to the extent of the diffuse emission in CGs found in Ponman et al. (1996) and Mulchaey et al. (2003) (noting the exceptions of HCGs 62 and 90). All data were taken in VFAINT mode except HCG 16, HCG 62 (ObsID 921), and HCG 92 (ObsID 789), which were in FAINT mode. Due to the design of the high-resolution mirror assembly, observations with the Chandra ACIS camera have superior spatial resolution $\left(\sim 0^{\prime \prime} .5\right.$ FWHM) compared to other instruments such as the ROSAT PSPC $\left(\sim 25^{\prime \prime}\right)$ and XMM EPIC $\left(\sim 6^{\prime \prime}\right)$, allowing for more robust removal of point sources that contaminate the diffuse emission and can lead to incorrect estimations of hot gas properties. Table 1 lists the observations of the CGs included in our sample.

The data were reprocessed beginning with the level 1 events file. We used the CIAO tool acis_reprocess_events with standard event filtering and VFAINT background cleaning (when appropriate) to produce the level 2 events file; however, we omitted the pixel randomization step in the event file processing to prevent degradation of the spatial resolution. The pixel randomization introduces a 0 '.5 random resampling of the event positions on the detector. This procedure is normally performed to mitigate the effects of aliasing in observations $\lesssim 2 \mathrm{ks}$, however, the observations included in our sample are substantially longer than this limit, and therefore the pixel randomization is not required. The pixel randomization has been shown to decrease the spatial resolution of the observations by $\sim 12 \%$ (e.g., Chartas et al. 2002). High spatial resolution is useful for proper removal of point sources that may be embedded within or projected onto any diffuse emission present in the groups.

Because the diffuse intragroup emission is typically weak and there is no robust method to determine the full radial extent of it from the data, for most targets we did not perform a spectral extraction in an annulus surrounding the targets to determine the soft X-ray background (SXRB) level. Instead, we subtracted the instrumental background using the stowed
ACIS background files appropriate to each observation (as in White et al. 2003). The stowed ACIS background files ${ }^{8}$ were obtained by sliding ACIS out of view of the sky and away from the external calibration source to avoid spectral-line contamination. All stowed background observations were taken after 2002 in VFAINT mode with gain corrections applied. Following procedures outlined in the ACIS Extract manual (Broos et al. 2010), we removed the "Clean55" bit from the event lists for subtraction from data taken in FAINT mode and renormalized the background files to the data using the particle background in the range 9-12 keV. The stowed background files include emission from the high-energy particle background $(E \gtrsim 2 \mathrm{keV})$, but not the SXRB $(E \lesssim 2 \mathrm{keV})$, therefore we expect contamination from the SXRB at energies $<2 \mathrm{keV}$. Table 1 lists the total counts extracted from and exposure times of the stowed background data for each group.

We used the latest version of ACIS Extract to create exposure maps, model point-source point spread functions (PSFs) and excise them from the observations, and extract spectral information (see Section 3). The energy used to create the exposure maps was the median event energy over the range $0.5-3.0 \mathrm{keV}$, chosen to coincide with the peak of any soft diffuse emission in the data.

\section{SPECTRAL EXTRACTION AND MODELING}

\subsection{Point Source Detection and Removal}

We used the CIAO Mexican-hat wavelet detection routine wavdetect (Freeman et al. 2002) to search for point sources in the field of view down to a source-significance threshold of $10^{-5}$. Detections were examined by eye to remove spurious sources (e.g., those with an axis length $\lesssim 1$ pixel). We then used ACIS Extract to model the PSF of the sources with MARX prior to excising the point sources from the events files. The excised regions correspond to 1.1 times the $99 \%$ encircled energy radius.

\footnotetext{
8 This brief description of the stowed ACIS background is based on the work of Maxim Markevitch; further details are available at http://cxc.harvard.edu/contrib/maxim/stowed/.
} 
Table 2

Best-fit Spectral Model Parameters

\begin{tabular}{|c|c|c|c|c|c|c|}
\hline $\mathrm{HCG}^{\mathrm{a}}$ & $\begin{array}{c}\mathrm{H} \mathrm{I} \\
\left(10^{20} \mathrm{~cm}^{-2}\right)\end{array}$ & $\begin{array}{c}k T \\
(\mathrm{keV})\end{array}$ & $Z / Z_{\odot}$ & $\begin{array}{c}A_{\mathrm{MEKAL}} \\
\left(10^{-4} \mathrm{~cm}^{-5}\right)\end{array}$ & $\begin{array}{c}\log _{10}\left(L_{X}\right) \\
\left(\operatorname{erg~s}^{-1}\right)\end{array}$ & $\chi^{2} /$ dof \\
\hline $7^{b}$ & 1.97 & 0.6 & 0.5 & $<0.29$ & $<40.35$ & $\cdots$ \\
\hline 16 & 2.56 & $0.65 \pm 0.06$ & 0.16 & $3.90_{-1.96}^{+2.20}$ & $41.10_{-0.30}^{+0.19}$ & $40.43 / 47$ \\
\hline $22^{b}$ & 4.26 & 0.6 & 0.5 & $<0.21$ & $<39.84$ & $\ldots$ \\
\hline 31 & 5.70 & $0.65_{-0.31}^{+0.18}$ & 0.05 & $1.16_{-0.65}^{+3.48}$ & $40.54_{-0.36}^{+0.60}$ & $31.43 / 40$ \\
\hline 42 & 4.11 & $0.72 \pm 0.01$ & 0.55 & $8.38_{-1.75}^{+1.76}$ & $41.82_{-0.10}^{+0.08}$ & $196.48 / 114$ \\
\hline 59 & 2.64 & $0.29_{-0.05}^{+0.41}$ & 17.53 & $0.01 \pm 0.004$ & $40.08_{-0.16}^{+0.12}$ & $55.84 / 61$ \\
\hline 62 (921) cool & 3.32 & $0.71 \pm 0.01$ & 16.68 & $0.50_{-0.49}^{+1.23}$ & $41.88_{-1.81}^{+0.54}$ & $319.10 / 281$ \\
\hline 62 (921) hot & 3.32 & $1.20 \pm 0.04$ & 0.48 & $1.46_{-1.16}^{+1.47}$ & $42.00 \pm 0.04$ & $319.10 / 281$ \\
\hline 62 (921) total & & & & & $42.23_{-0.26}^{+0.34}$ & \\
\hline $62(10462+10874) \mathrm{cool}$ & 3.32 & $0.71 \pm 0.01$ & 16.68 & $0.46_{-0.45}^{+1.12}$ & $41.84_{-1.81}^{+0.54}$ & $319.10 / 281$ \\
\hline $62(10462+10874)$ hot & 3.32 & $1.20 \pm 0.04$ & 0.48 & $1.48_{-1.08}^{+1.43}$ & $42.01_{-0.03}^{+0.04}$ & $319.10 / 281$ \\
\hline 62 average total & & & & & $42.72_{-0.26}^{+0.33}$ & \\
\hline $90 \mathrm{BCD}$ & 2.02 & $0.66 \pm 0.03$ & 0.54 & $2.66_{-1.50}^{+1.59}$ & $40.79_{-0.36}^{+0.20}$ & $121.75 / 119$ \\
\hline $92(789)$ & 6.16 & $0.63 \pm 0.02$ & 0.14 & $6.25_{-0.99}^{+1.08}$ & $41.77_{-0.08}^{+0.07}$ & $357.33 / 220$ \\
\hline 92 (7924) & 6.16 & $0.63 \pm 0.02$ & 0.14 & $5.81_{-1.12}^{+1.28}$ & $41.73_{-0.10}^{+0.09}$ & $357.33 / 220$ \\
\hline 92 average & & & & & $41.76 \pm 0.07$ & \\
\hline
\end{tabular}

Notes.

${ }^{a}$ For groups with spectra that were fitted simultaneously rather than merged, we list the corresponding ObsIDs in parentheses.

b No detection, model temperature and abundance fixed at $0.6 \mathrm{keV}$ and $0.5 Z_{\odot}$, respectively.

For extraction of spectra, we did not interpolate over the holes created by excising the point sources to avoid making statistical assumptions concerning the gas. The area of diffuse emission on the sky lost due to point-source removal was typically less than $1 \%$ of the total extraction region. We defer the examination of the point sources in our sample to P. Tzanavaris et al. (in preparation).

\subsection{Extraction of the Diffuse Emission}

Extraction regions were centered on the apparent centers of the CG galaxy distributions with shapes and sizes chosen to best cover all of the main group members on the S3 chip (I0 for HCG 90), including evident diffuse emission in the level 2 events files. The extraction regions were either circular or elliptical in shape, except in the cases of HCGs 16 and 22. In these groups, the regions were rectangular and placed at a position angle to include the main galaxies and any known neutral gas. The area of each extraction region can be found in Table 1, while additional information is located in the Appendix. Note that in the case of HCG 22, the extraction region includes a background pair of galaxies to the southeast of the group center. As this group was classified as a non-detection, we set an upper limit on the diffuse $\mathrm{X}$-ray luminosity using the method outlined below. Therefore, the inclusion of this pair in the extraction region has negligible impact on the results.

Prior to model fitting in XSPEC (Arnaud 1996), the extracted spectra were rebinned such that each pulse height amplitude (PHA) bin contained a minimum of 20 counts. This ensured statistically valid results when fitting with $\chi^{2}$ statistics. In cases where the ACIS instrumental response did not change significantly between observations (i.e., multiple ObsIDs within an observing cycle), we merged the on-source spectra to reduce the relative error in each PHA bin. When merging the spectra was not possible due to the changes in the instrument response, we simultaneously fit the model to each spectrum with the temperature and metal abundances linked between different
ObsIDs; however, the model normalization was allowed to vary freely.

A detection was defined to have $\mathrm{S} / \mathrm{N} \geqslant 3$. The noise of the spectra was determined to be $\sigma=\left[\mathrm{SB}+\left(A_{s} t_{s} / A_{b} t_{b}\right) B\right]^{1 / 2}$, where SB is the total counts in the source before background subtraction, $B$ is the number of counts in the background, $A$ is the area of the extraction region, $t$ is the integration time, and the subscripts $s$ and $b$ represent the science and background observations, respectively. When using the stowed background, the background counts were scaled to the 9-12 keV count rates in the science observations prior to estimating the noise. This scaling has the effect of normalizing the shape of the stowed background spectrum to that of the source in a range of energies dominated by the particle background (Hickox \& Markevitch 2006). When no diffuse emission was detected, we put an upper limit on the luminosity of the intragroup medium by fixing the temperature and metal abundance of the plasma to reasonable values of $0.6 \mathrm{keV}$ and $0.5 Z_{\odot}$, respectively. We then adjusted the normalization to match the sum of the observed count rate and the $1 \sigma$ noise estimate.

In all cases when diffuse X-ray emission was not detected, the energy range over which the count rates were evaluated had to be restricted to $0.7-1.7 \mathrm{keV}$ due to oversubtraction of the stowed background between 1.7 and $2 \mathrm{keV}$. Bolometric X-ray luminosities were then computed in the same manner as for groups with detected emission (see below).

\subsection{Spectral Model Fitting}

We fit a combination of optically thin plasma and Galactic photoelectric absorption models for each of the groups. The best-fitting models are presented in Table 2. The first model component, an optically thin plasma, was modeled using the MEKAL model (Mewe et al. 1985, 1986; Kaastra 1992, 1993; Liedahl et al. 1995; Kaastra \& Liedahl 1995) with the adopted ionization balance taken from Arnaud \& Rothenflug (1985) and Arnaud \& Raymond (1992). We fixed the hydrogen number 
density at a reasonable value of $1 \mathrm{~cm}^{-3}$ and allowed the remaining parameters to vary freely. While the metallicity parameter was allowed to vary, these values are poorly constrained by the available data, and we only report them here to describe the best-fitting models to the observations. We calculated the model at all temperatures rather than interpolating it from a precalculated table while fitting the data. Other than the plasma temperature, we also report the normalization used in calculating the X-ray luminosity. We note that because the gas is often associated with individual galaxies rather than a single distribution permeating the intragroup medium (see Section 4.1), it is likely multi-temperature; however, we find that the singletemperature plasma model fits the extracted spectra well, except in the case of HCG 62 for which a two-temperature plasma results in a better fit. Note that HCG 62 has the most counts in the background-subtracted data compared to any other observation in this study-specifically, a factor of $\sim 7$ more counts in the merged ObsIDs 10462 and 10874 compared to HCG 90, which has the next most counts. For other targets, our ability to isolate the multiple spectral components that likely make up the diffuse $\mathrm{X}$-ray emission is limited by the number of counts; therefore, it is probable that we cannot detect spectral complexity in our data (e.g., multi-temperature plasmas) given the limited numbers of counts.

Photoelectric absorption was modeled using the TuebingenBoulder interstellar medium absorption model from Wilms et al. (2000). The only model parameter, the H I column density along the line of sight, was fixed at the value determined using the HEASoft tool $\mathrm{nH}$ to compute the weighted mean of $\mathrm{H} \mathrm{I}$ in a cone centered on the source and with a radius of $1^{\circ}$. Following the recommendation of the $\mathrm{nH}$ manual, ${ }^{9}$ we use the $\mathrm{H}$ I values from Kalberla et al. (2005).

Abundances and depletion values for the model (used in both the absorption and emission components) were taken from Lodders (2003; the most recent abundances available in XSPEC) rather than the default from Anders \& Grevesse (1989). Both abundance tables use solar photospheric line and CI chondrite analyses to determine the relative amounts of each element. We found that the values from Lodders (2003) consistently performed better at fitting emission-line features in the spectra, particularly in observations with significant numbers of counts (e.g., HCG 62).

Because Chandra only has significant response over the energy range $0.3-8 \mathrm{keV}$, we computed the bolometric X-ray luminosity $L_{X}$ using a dummy response over the energy range 0.01-100 keV logarithmically divided into 5000 energy bins. We used $3 \mathrm{~K}$ cosmic microwave background (CMB) adjusted velocities (Fixsen et al. 1996) from the NASA Extragalactic Database (NED), which adjusts the velocities for the observed dipole anisotropy in the $\mathrm{CMB}$, to determine the distances to the sources for luminosity calculations. Parameters of interest, specifically the temperature and luminosity of the plasma (and by necessity, the model normalization), are reported with $90 \%$ confidence error bars. For simultaneously fit spectra, we report the average luminosity (weighted by the number of counts) determined from the model fits. Note that in the case of HCG 59, the peak in energy of the X-ray emission is very poorly constrained; therefore we fix the temperature to the best-fitting value prior to determining the error in the model normalization. Furthermore, we do not consider the best-fitting value to be representative of the real temperature and simply report it as

\footnotetext{
9 http://heasarc.nasa.gov/Tools/w3nh_help.html\#comparison
}

$<1 \mathrm{keV}$, however we do use the temperature result of the model fit for qualitative purposes in the figures below. We present the temperatures and luminosities derived from the fitted models, as well as goodness-of-fit estimations, in Table 2.

\section{RESULTS AND DISCUSSION}

We identify hot diffuse gas in seven of nine groups (HCGs 16, $31,42,59,62,90$, and 92) in our X-ray sample. Of the detected groups, diffuse emission in HCG 31 has not been reported in previous studies. Both of the two groups without detections, HCGs $7^{10}$ and 22, have low to negligible star formation without much evidence of strong tidal interactions in the past few Gyr (e.g., Konstantopoulos et al. 2010). In the detected groups, the temperatures are all fairly similar (0.6-0.72 keV, not including the hot component of HCG 62 or the anomalously low temperature of HCG 59, which is very poorly constrained), while the range in X-ray luminosities spans $10^{40.37}-10^{42.18} \mathrm{erg} \mathrm{s}^{-1}$. The morphology of the hot gas ranges from isolated around individual group members to common X-ray halos (e.g., HCGs 16 and 62, respectively), as well as gas bridges connecting galaxies (HCGs 59 and 90; see Section 4.1). In Figures 1-7, we plot all values derived from model fits for multiple observations that could not be merged prior to fitting.

Table 3 lists relevant information about each of the groups in our sample. Included in the table are the group redshift and $3 \mathrm{~K} \mathrm{CMB}$ velocity, the calculated velocity dispersion, number of main group members, the number of galaxies used in the velocity dispersion calculation, the number of E/S0-type galaxies that are not considered dwarf members, the SSFR from Tzanavaris et al. (2010), the total H I mass from Verdes-Montenegro et al. (2001) and Borthakur et al. (2010), the dynamical mass determined from the group velocity dispersion and mean two galaxy separation taken from Hickson et al. (1992), and the group Hi evolutionary type defined by Johnson et al. (2007; see Section 4.3 for a full description). We will continually refer back to these data throughout the figures and discussion that follow.

The nine groups in our sample were also observed with ROSAT and the results presented in Ponman et al. (1996). We compare our temperatures and luminosities derived from fitting the extracted spectra from the Chandra data with those also detected by Ponman et al. (1996; i.e., HCGs 16, 42, 62, 90 and 92) in Table 4. Rather than directly compare the luminosities, we calculate the fluxes of the sources, which removes the dependence of the assumed cosmology. The redshifts used in the flux calculation for the ROSAT data are the same as those listed in the redshift column of Table 3 . Note that Ponman et al. (1996) give errors in the luminosity and temperature at the $1 \sigma$ level. For the temperature in HCG 62, we use the luminosityweighted average of the two plasma temperatures, while the fluxes in HCGs 62 and 92 are based on the average of the luminosities weighted by total counts in the different Chandra ObsIDs, therefore the percent errors on these values seem quite large because of the compounding of errors from multiple measurements. In all cases, the flux of the X-ray emission from

\footnotetext{
10 A deeper $49 \mathrm{ks} X M M$ observation (PI: Belsole) of this group exists, but no results have been published at the time of this writing. A brief inspection of the data shows several dozen point sources and large-scale diffuse emission across the field of view of the EPIC camera ( $\sim 5$ times the angular size of HCG 7$)$. The sensitivity of $X M M$ to cool, extended gas complicates the interpretation of this emission as either related to the group or part of the SXRB. Results from this and other XMM data will be included in subsequent papers.
} 

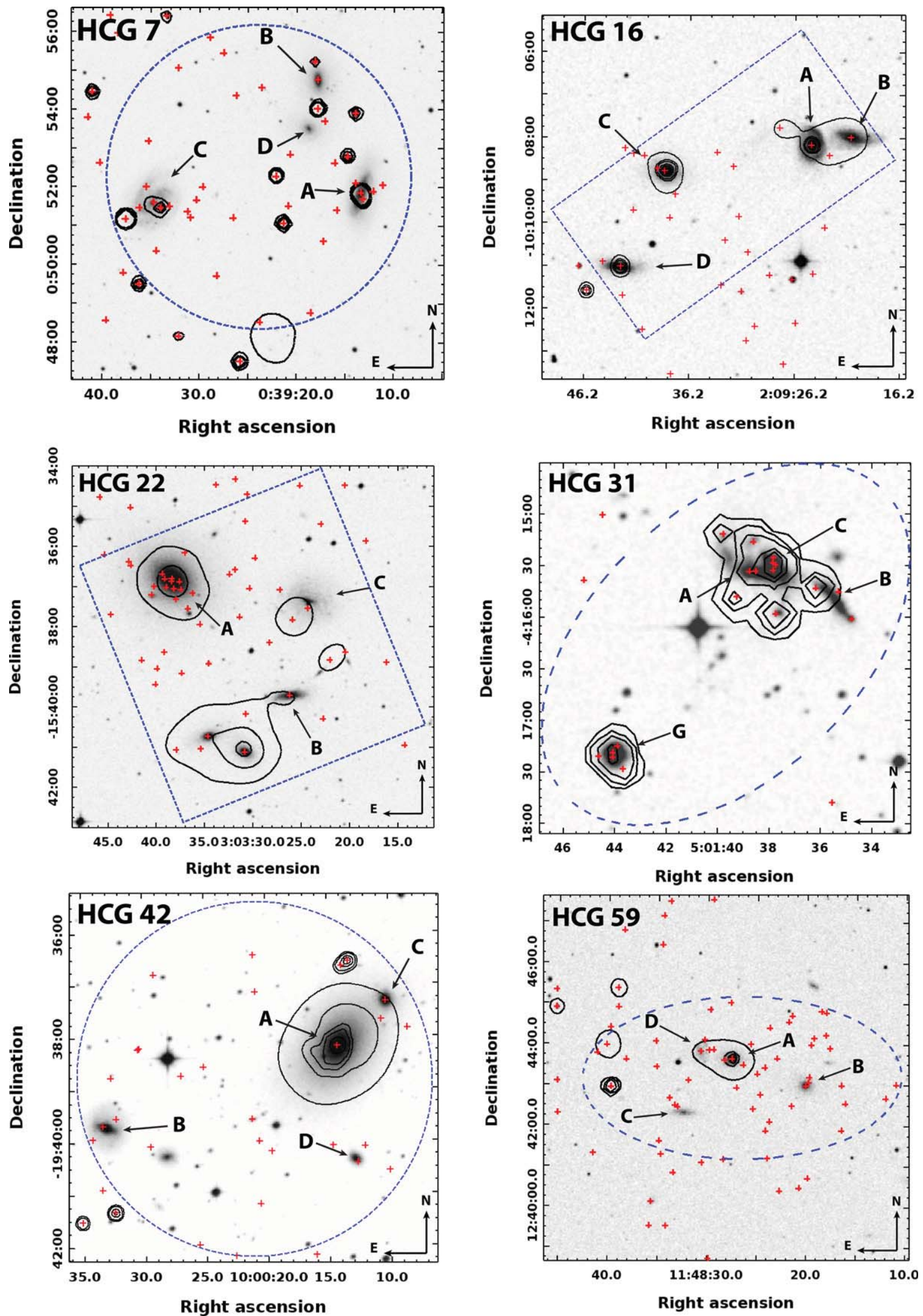

Figure 1. Smoothed 0.5-2.0 keV X-ray contours of groups in our sample. The event files were smoothed using the CIAO task csmooth with the minimum significance set to $3 \sigma$ above the stowed background scaled by the $9-12 \mathrm{keV}$ count rate. The minimum and maximum smoothing scales used were 2 and 10 pixels, respectively. In all images, the dashed blue line and red plus symbols correspond to the extraction region used (see the Appendix) and the locations of the X-ray point sources that were excised from the analysis (but retained in the smoothed X-ray data), respectively. The optical images are DSS POSS2 survey red filter data. Contour levels in counts follow in the captions for each group: HCG 7: 0.1, 0.2, 0.3; HCG 16: 0.1, 0.5, 1, 3, 5, 10; HCG 22: 0.1, 0.2, 0.3; HCG 31: 0.2, 0.5, 1, 3, 5; HCG 42: 0.5, 1, 3, 5, 10; HCG 59: 0.2, 0.5, 1, 3, 5, 10; HCG 62: 3, 6, 10, 15, 30, 50; HCG 90: ; HCG 92: 0.5, 1, 2, 5, 10.

(A color version of this figure is available in the online journal.) 

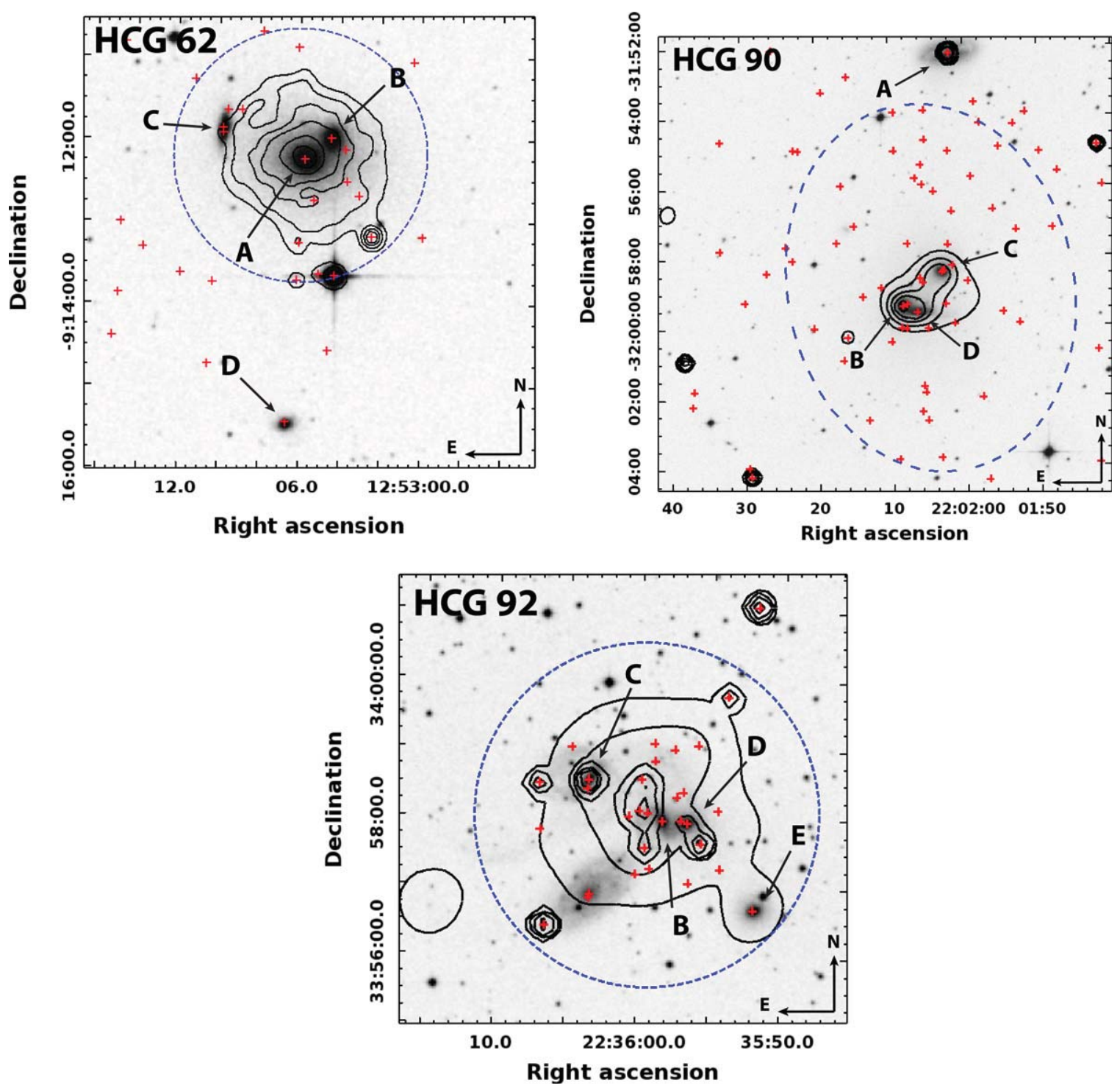

Figure 1. (Continued)

the ROSAT data is found to be brighter in the Ponman et al. (1996) study. When comparing the temperatures we determined against those from Ponman et al. (1996), we find that HCGs 62 and 90 are consistent between the two studies, while HCG 16 is significantly hotter, and HCGs 42 and 92 are significantly cooler than previously reported. The mean temperature of the groups detected in both studies is 0.70 and $0.72 \mathrm{keV}$ with standard deviations of 0.25 and $0.14 \mathrm{keV}$ in Ponman et al. (1996) and this study, respectively. The temperature discrepancies are likely due to the difference in the PSF of the ROSAT PSPC instrument compared to the Chandra ACIS camera. Specifically, the detection and subtraction of point sources in the diffuse emission is much more robust using Chandra data, and point sources contaminating the extracted spectrum would alter the peak of the emission leading to an incorrect estimation of the hot gas temperature. We also note that Ponman et al. (1996) attempted to subtract the diffuse X-ray contribution of the individual group members and interpolate over the "holes." This, combined with distinctions in the extraction regions and the responses of the ROSAT PSPC and Chandra ACIS instruments, may explain the differences in the results.

\subsection{Gas Distribution and Morphology}

Figure 1 shows the smoothed diffuse X-ray emission in the CGs included in our study. To construct the X-ray maps, we smoothed the level 2 events files in the range of $0.5-2 \mathrm{keV}$ using the adaptive smoothing csmooth algorithm. Pixels with $\mathrm{S} / \mathrm{N}>3$ above the stowed background were smoothed with a Gaussian kernel with smoothing scales between 2 and 10 pixels. From the figures, we note that the hot gas in CGs exhibits varied morphologies including small halos around one or several galaxies, plumes centered on particular group members, tidal bridges, and large common halos encompassing most of the group.

The maps were qualitatively assessed by eye to categorize the observed X-ray emission as either associated with the environment or the individual group members. Of the nine CGs included in our study, we do not detect diffuse emission from HCGs 7 and 22. From those groups detected with Chandra, only HCG 62 has emission that permeates the IGM, while HCG 42 has a bright X-ray halo centered on the brightest group galaxy (i.e., 42A). We consider these two groups to be indicative of 
Table 3

Properties of Sample HCGs

\begin{tabular}{|c|c|c|c|c|c|c|c|c|c|c|c|c|}
\hline \multirow[t]{2}{*}{$\mathrm{HCG}$} & \multirow[t]{2}{*}{ Redshift } & \multirow{2}{*}{$\begin{array}{c}v_{\mathrm{CMB}}{ }^{\mathrm{a}} \\
\left(\mathrm{km} \mathrm{s}^{-1}\right)\end{array}$} & \multirow{2}{*}{$\begin{array}{l}\text { Dispersion } \\
\left(\mathrm{km} \mathrm{s}^{-1}\right)\end{array}$} & \multirow{2}{*}{$\begin{array}{c}\text { Velocity } \\
\text { References }\end{array}$} & \multicolumn{3}{|c|}{ Number of Galaxies } & \multirow{2}{*}{$\begin{array}{c}\mathrm{SSFR}^{\mathrm{c}} \\
\left(10^{-11} \mathrm{yr}^{-1}\right)\end{array}$} & \multirow{2}{*}{$\begin{array}{c}\log _{10}\left(M_{\text {dyn }}\right) \\
\left(M_{\odot}\right)\end{array}$} & \multirow{2}{*}{$\begin{array}{c}\log _{10}\left(M_{\mathrm{HI}}\right) \\
\left(M_{\odot}\right)\end{array}$} & \multirow{2}{*}{$\begin{array}{l}\log _{10}\left(M_{\mathrm{HI}_{\mathrm{I}}}\right) / \\
\log _{10}\left(M_{\mathrm{dyn}}\right)^{\mathrm{d}}\end{array}$} & \multirow{2}{*}{$\begin{array}{c}\text { Evo } \\
\text { Type }^{\mathrm{e}}\end{array}$} \\
\hline & & & & & Main & Dyn $^{b}$ & $\overline{\mathrm{E} / \mathrm{S} 0}$ & & & & & \\
\hline 7 & 0.0141 & 3885 & $129_{-8}^{+9}$ & $1-4$ & 4 & 5 & 1 & $8.79 \pm 0.75$ & 12.10 & 9.76 & 0.81 & II A \\
\hline 16 & 0.0132 & 3706 & $84_{-4}^{+5}$ & $4-7$ & 5 & 7 & 0 & $56.19 \pm 7.13$ & 11.73 & $>10.42$ & $>0.89$ & II B \\
\hline 22 & 0.0090 & 2522 & $37_{-8}^{+13}$ & $8-11$ & 3 & 4 & 1 & $6.24 \pm 0.95$ & 10.78 & 9.13 & 0.85 & II A \\
\hline 31 & 0.0135 & 4026 & $56_{-7}^{+5}$ & $1,12-14$ & 4 & 8 & 0 & $89.63 \pm 15.46$ & 10.64 & 10.27 & 0.97 & I B \\
\hline 42 & 0.0133 & 4332 & $273 \pm 12$ & 15,16 & 4 & 38 & 4 & $0.74 \pm 0.11$ & 12.75 & 9.40 & 0.74 & III B \\
\hline $59^{f}$ & 0.0135 & 4392 & $208 \pm 12$ & $17-20$ & 4 & 8 & 1 & $51.82 \pm 11.04$ & 12.19 & 9.49 & 0.78 & III \\
\hline $62^{f}$ & 0.0137 & 4443 & $398 \pm 7$ & $\begin{array}{l}7,11,15 \\
16,21-23\end{array}$ & 4 & 62 & 4 & $0.92 \pm 0.19$ & 12.86 & $<9.06$ & $<0.70$ & III \\
\hline 90 & 0.0088 & 2364 & $177_{-9}^{+8}$ & 8,15 & 4 & 16 & 2 & $\cdots$ & 12.19 & 8.70 & 0.71 & III A \\
\hline 92 & 0.0215 & 6119 & $343 \pm 6$ & $1,12,20$ & 4 & 4 & 2 & $\cdots$ & 12.75 & 10.23 & 0.80 & II B \\
\hline
\end{tabular}

Notes.

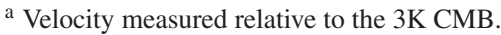

b Number of galaxies used in velocity dispersion calculation.

c Total UV+24 $\mu \mathrm{m}$ specific star formation rates by Tzanavaris et al. (2010) with corrections from P. Tzanavaris (2012, private communication).

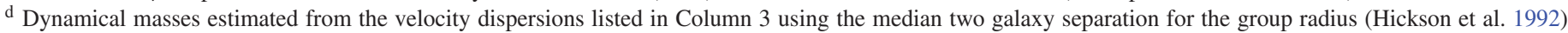
corrected to our cosmology. The most recent H i masses were taken from Verdes-Montenegro et al. (2001) and Borthakur et al. (2010).

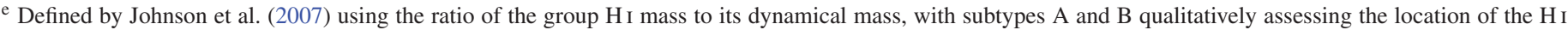
gas (localized to the group members or spread throughout the group, respectively; Konstantopoulos et al. 2010).

${ }^{\mathrm{f}}$ No $\mathrm{H}$ i imaging data available to estimate subtypes in the evolutionary classification.

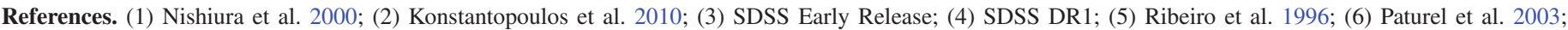

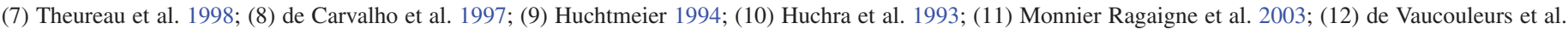

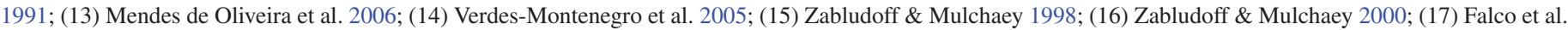
1999; (18) SDSS DR4; (19) SDSS DR8; (20) Hickson et al. 1992; (21) da Costa et al. 1998; (22) HIPASS Final Catalog; (23) Jones et al. 2009.

Table 4

Comparison of Chandra and ROSAT Measurements

\begin{tabular}{|c|c|c|c|c|c|c|}
\hline HCG & $\begin{array}{l}T_{X, \mathrm{P} 96} \\
(\mathrm{keV})\end{array}$ & $\begin{array}{c}T_{X} \\
(\mathrm{keV})\end{array}$ & $\begin{array}{c}\Delta T_{X} \\
(\mathrm{keV})\end{array}$ & $\begin{array}{c}F_{X, \mathrm{P} 96^{\mathrm{a}}} \\
\left(10^{-12} \mathrm{erg} \mathrm{s}^{-1} \mathrm{~cm}^{-2}\right)\end{array}$ & $\begin{array}{c}F_{X}{ }^{\mathrm{a}} \\
\left(10^{-12} \operatorname{erg~s}^{-1} \mathrm{~cm}^{-2}\right)\end{array}$ & $\begin{array}{c}\Delta F_{X} \\
\left(10^{-12} \mathrm{erg} \mathrm{s}^{-1} \mathrm{~cm}^{-2}\right)\end{array}$ \\
\hline 16 & $0.30 \pm 0.05$ & $0.65 \pm 0.06$ & $-0.35 \pm 0.08$ & $7.97_{-1.03}^{+1.18}$ & $1.98_{-0.99}^{+1.34}$ & $5.99_{-1.43}^{+1.78}$ \\
\hline 42 & $0.82 \pm 0.03$ & $0.72 \pm 0.01$ & $0.10 \pm 0.03$ & $23.71_{-1.07}^{+1.12}$ & $20.74_{-4.34}^{+4.38}$ & $2.97_{-4.47}^{+4.52}$ \\
\hline $62^{\mathrm{b}}$ & $0.96 \pm 0.04$ & $0.99 \pm 0.04$ & $-0.03 \pm 0.06$ & $169.48_{-11.31}^{+12.12}$ & $158.30_{-70.78}^{+182.19}$ & $11.18_{-71.68}^{+182.59}$ \\
\hline 90 & $0.68 \pm 0.12$ & $0.66 \pm 0.03$ & $0.02 \pm 0.12$ & $11.34_{-2.12}^{+2.61}$ & $4.47_{-2.53}^{+2.67}$ & $6.87_{-3.30}^{+3.73}$ \\
\hline 92 & $0.75 \pm 0.08$ & $0.63 \pm 0.02$ & $0.12 \pm 0.08$ & $9.04_{-0.80}^{+0.87}$ & $6.95_{-1.09}^{+1.20}$ & $2.09_{-1.35}^{+1.48}$ \\
\hline
\end{tabular}

Notes. P96: Ponman et al. (1996).

a Converted from the bolometric luminosity and adjusted for differences in cosmology.

b The values listed for this study are the flux-weighted average temperature and total flux of the two plasma components in HCG 62 .

systems with X-ray emission similar in morphology to clusters. The other groups, HCGs 16, 31, 59, 90, and 92 (excepting the shock front in the case of HCG 92), are those in which the hot gas is associated with the individual galaxies. Mulchaey (2000) noted that previous X-ray telescopes did not have the requisite spatial resolution to separate the intragroup gas from the galaxy-linked emission; however, the resolution of Chandra is well suited to this task. Furthermore, we join Tamburri et al. (2012), who presented a Chandra study of HCG 79 (also known as "Seyfert's Sextet"), in speculating that perhaps many CGs exhibit galaxy-linked X-ray emission rather than a hot intragroup medium associated with the environment. Throughout the remainder of the paper, unless explicitly stated, we discuss the galaxy- and environment-linked diffuse X-ray emission together as they fit into a larger picture of group evolution, i.e., from dynamically unevolved systems with X-ray emission confined to the galaxies to more evolved systems with a single X-ray halo. We stress that it is difficult to disentangle the galaxy- and group-linked emission in the data as this distinction is not always clear, as in the emission surrounding HCG 42A.
In the specific case of HCG 16, the group contains two members ( $\mathrm{C}$ and $\mathrm{D})$ with hot gas plumes that are coincident with extended $\mathrm{H} \alpha$ emission (see Figure 2). Both galaxies show clear signs of recent interactions (e.g., disturbed velocity fields; gas/stellar disk misalignments; Mendes de Oliveira et al. 1998; a common H I envelope surrounding the group; Verdes-Montenegro et al. 2001) and are strong starbursts with SFRs of 14 and $17 M_{\odot} \mathrm{yr}^{-1}$, respectively, and SSFRs of $(2-3) \times 10^{-10} \mathrm{yr}^{-1}$ (Ribeiro et al. 1996; Tzanavaris et al. 2010). Rich et al. (2010) used integral field spectroscopy to demonstrate that the motions of the gas and the optical emission-line profiles of $16 \mathrm{D}$ are indicative of an M82-like superwind due to the intense starburst within the galaxy, and that 16D and M82 share some similar properties (e.g., metallicity, $L_{\mathrm{IR}}, L_{\mathrm{H} \alpha}$ ). Based on its high SFR and extended emission-line gas, 16C may represent a second M82-like starburst in the HCG 16 group, possibly triggered by a recent $(\lesssim 1 \mathrm{Gyr})$ tidal interaction with $16 \mathrm{~A}$, similar to the encounter between M81 and M82 220 Myr ago (Gottesman \& Weliachew 1977; Konstantopoulos et al. 2009). Furthermore, Jeltema et al. (2008) found evidence for a weak 


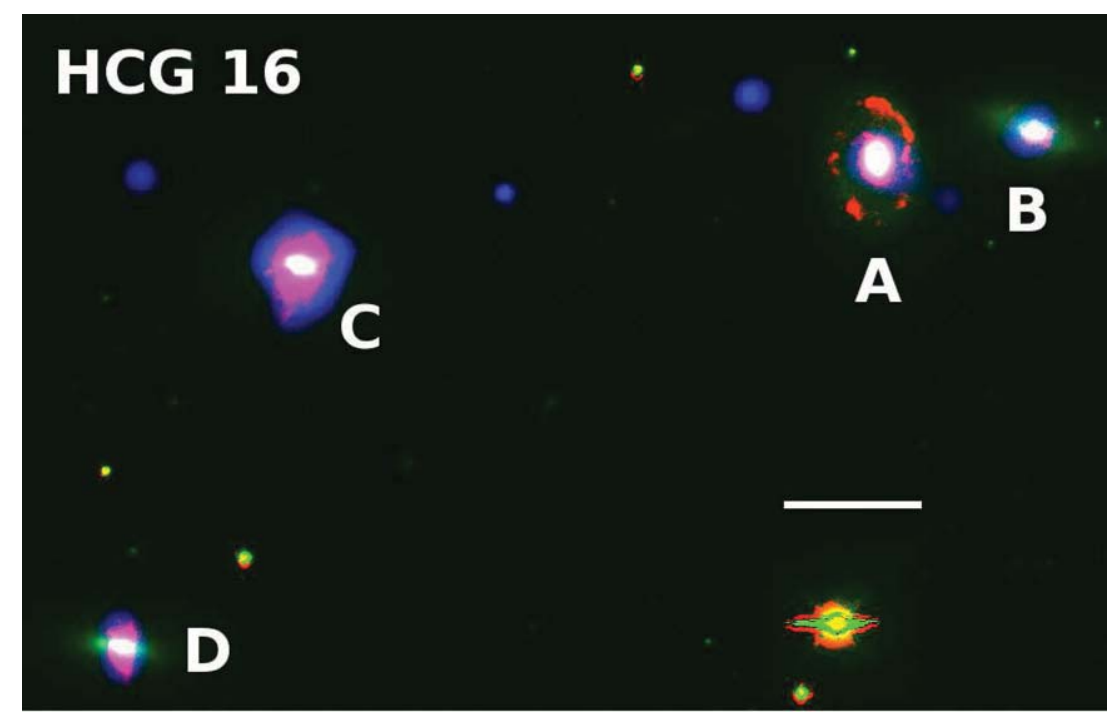

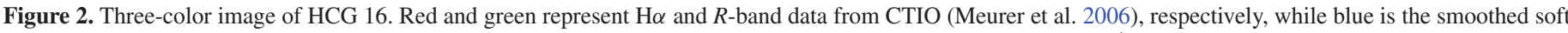

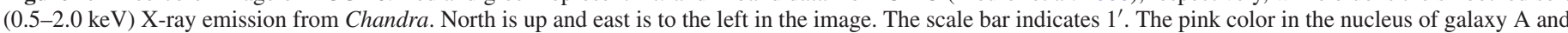

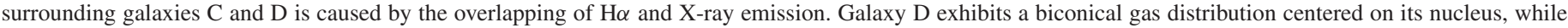
ionized gas surrounds galaxy $\mathrm{C}$ with an elongation along the north-to-south axis.

soft X-ray bridge connecting galaxies $16 \mathrm{~A}$ and $\mathrm{B}$ indicating a recent tidal interaction between these group members as well.

Further examining the morphology of the hot gas and its relation to the galaxies in the CGs, we find that the gas in low dynamical mass $\left(\lesssim 10^{12.5} M_{\odot}\right)$ groups with low-velocity dispersions $\left(<250 \mathrm{~km} \mathrm{~s}^{-1}\right)$ is concentrated around the individual group members, while gas in relatively higher mass systems with larger velocity dispersions begins to resemble a common envelope.

\subsection{The $L_{X}-T$ and $L_{X}-\sigma$ Relations Revisited}

Previous work has examined the relationships between the $\mathrm{X}$-ray luminosity and both the plasma temperature and the cluster/group velocity dispersion in systems of galaxies (e.g., Ponman et al. 1996; Mulchaey \& Zabludoff 1998; Wu et al. 1999; Helsdon \& Ponman 2000; Osmond \& Ponman 2004; Mulchaey et al. 2003). The cluster data have been found to show very little scatter in $L_{X}-T$ and $L_{X}-\sigma$ space. However, groups, with fainter X-ray luminosities, have been found to exhibit a larger spread potentially due in part to uncertainties in the measurements, or because they are not virialized systems (see below). We merge the cluster data from Wu et al. (1999) and Zhang et al. (2011) for comparison to the CGs. Prior to merging, we first adjusted the $\mathrm{Wu}$ et al. (1999) data to our assumed cosmology, while Zhang et al. (2011) used the same cosmology as that assumed in this paper. When comparing the cluster data to the CGs in $L_{X}-T$ space, we only include the 176 clusters with uncertainties in both $L_{X}$ and temperature. This same criterion, with respect to velocity dispersion rather than temperature, is applied to the cluster data when comparing the groups and clusters in $L_{X}-\sigma$ space, resulting in 142 clusters in this sample. We note that the Zhang et al. (2011) clusters are measured to $r_{500}$ (i.e., the radius at which the average density falls to 500 times the critical density at that redshift), and the extraction regions used in this work vary between $26 \%$ and $69 \%$ of $r_{500}$. It is unclear to what radius the Wu et al. (1999) clusters were measured; however, the authors state that they used a $\beta$ model to correct all of their data to the same fraction of the virial radius.

Figure 3 shows the $L_{X}-T$ relationship for the groups and the clusters from Wu et al. (1999) and Zhang et al. (2011). Using linear regressions described by Akritas \& Bershady (1996), we fit the clusters in $L_{X}-T$ space not including the CGs. For this fit, we use the orthogonal distance regression (ODR) fitting method, which finds $\log _{10}\left(L_{X}\right)=(42.2 \pm 0.2)+(3.33 \pm 0.20) \log _{10}(T)$, where $L_{X}$ and $T$ are in units of erg s${ }^{-1}$ and $\mathrm{keV}$, respectively. Examining Figure 3, we find that HCGs 31, 42, 59, and 92 agree with the $L_{X}-T$ cluster relation within the errors. We note that HCG 62 lies slightly above the cluster relation (i.e., it is brighter for its temperature). HCGs 7, 16, 22, and 90 lie below the $L_{X}-T$ relation from the clusters.

Figure 4 shows the $L_{X}-\sigma$ relation for the groups in our sample. Velocity dispersions were calculated using the most accurate velocities available from NED for the CG members including additional known group members primarily from the large spectroscopic surveys of de Carvalho et al. (1997), Zabludoff \& Mulchaey (1998), and Zabludoff \& Mulchaey (2000) (see Table 3 for full velocity references). Velocity errors for individual group members were typically $\lesssim 30 \mathrm{~km} \mathrm{~s}^{-1}$. Again, we compare our CG data with the cluster data taken from Wu et al. (1999) and Zhang et al. (2011) using a linear least-squares fit. We chose this type of fit because the ODR method used in the $L_{X}-T$ diagram showed strong systematics in the residuals of the cluster data. The resulting best fit is $\log _{10}\left(L_{X}\right)=(33.7 \pm 0.6)+(3.70 \pm 0.21) \log _{10}(\sigma)$, where $L_{X}$ and $\sigma$ are in units of $\mathrm{erg} \mathrm{s}^{-1}$ and $\mathrm{km} \mathrm{s}^{-1}$. We find that two of our CGs (HCGs 16 and 31) agree with the $L_{X}-\sigma$ relation from the galaxy clusters within errors, while HCG 62 is similar to the clusters within the scatter. In the cases of HCGs 16 and 31, this agreement is likely coincidental because the $L_{X}-\sigma$ relation is predicted from the virial theorem to be $L_{X} \propto \sigma^{4}$, and the bulk of the hot gas in these systems is clearly due to star formation. We do note, however, that for groups in which dynamical processes are increasingly important in heating the gas (i.e., HCGs 42, 59, 62,90 , and 92), there does appear to be a monotonic increase in 


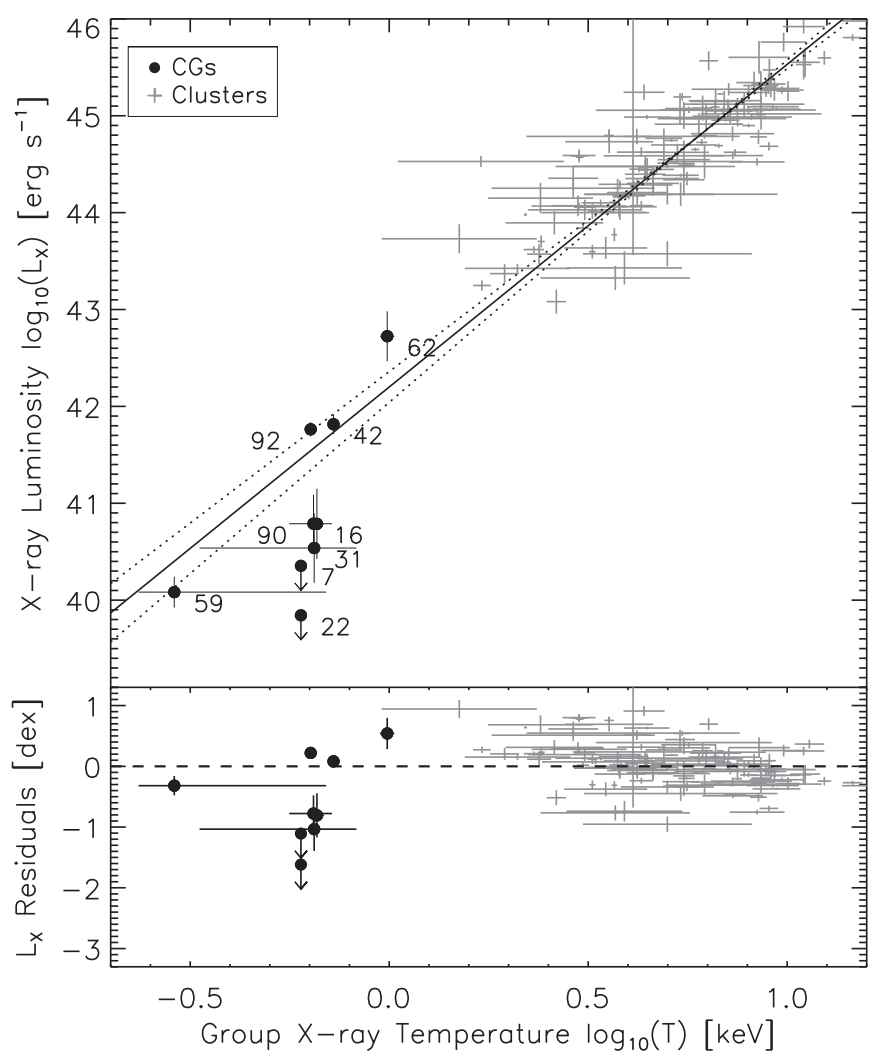

Figure 3. $L_{X}-T$ diagram for compact groups (black filled circles). The solid line indicates the best fit to the cluster data (gray plus symbols) from Wu et al. (1999) and Zhang et al. (2011) using an orthogonal distance regression (ODR) fit to 176 clusters, while the dashed lines represent the errors on the fit. We plot the two plasmas in HCG 62 as one data point using the luminosity-weighted average of the temperatures from Table 4 and the total X-ray luminosity. The temperature of HCG 59 is very uncertain due to the poorly defined peak in the $\mathrm{X}$-ray spectrum, and we conservatively estimate it to be $<1 \mathrm{keV}$; however, we include the best-fitting temperature from the MEKAL model here in the $L_{X}-T$ diagram for qualitative purposes. The best-fitting cluster relation agrees with HCGs 31, 42, 59, and 92 within the errors, while HCG 62 agrees lies within the scatter in the cluster data. We do note that most of the compact groups lie systematically below the cluster fit.

the X-ray luminosity with increasing velocity dispersion, albeit with some scatter.

Ponman et al. (1996) hypothesized that higher temperatures for a given $L_{X}$ than would be expected from the cluster $L_{X}-T$ relation for CGs could be a result of the injection of energy into the IGM from galactic winds, while Ponman et al. (1999) suggested that the observed deviation of the galaxy group $\mathrm{X}$-ray luminosities from the established cluster scaling relations could be explained by preheating of the IGM by supernovae. In the preheating model, heating of the gas in the IGM occurs early in the lifetimes of groups and similarly steepens the $L_{X}-T$ relation for these systems. Despite groups such as HCG 16, where star formation may play a more prominent role, the existence of X-ray brighter groups (i.e., HCGs 42) below the $L_{X}-T$ scaling relation for clusters indicates that galaxy winds alone are unlikely to explain the observed deviation. However, the observed dichotomy in X-ray gas morphology (i.e., galaxyversus environment-linked emission) may indicate that the low-mass, low-velocity dispersion systems are dynamically unevolved and have shallow potential wells that are unable to heat any neutral gas that has been liberated from the group members. In these systems, the role of individual galaxies may be more important in heating gas through local (e.g.,

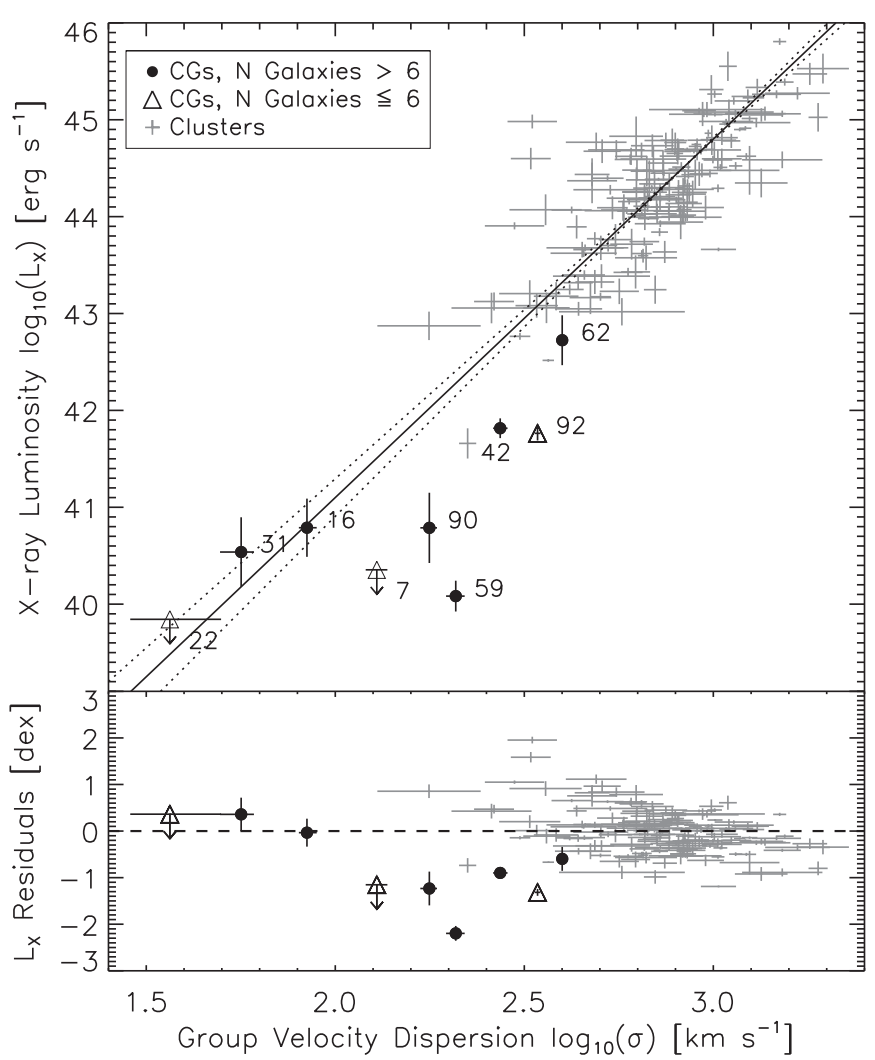

Figure 4. $L_{X}-\sigma$ diagram for compact groups. Due to the inaccuracy in the velocity dispersions for systems with a small number of components, we plot filled black circles to indicate groups for which six or more galaxies were used to compute the velocity dispersion, while open triangles represent groups below this threshold. The solid line indicates the best fit to the clusters using a linearleast-squares fit to the 142 clusters taken from Wu et al. (1999) and Zhang et al. (2011) (gray plus symbols), while the dashed lines represent the errors on the fit. The best-fitting cluster relation agrees with HCGs 16, 31, and 62, while HCGs 7, 42, 59, 90, and 92 lie below the fit. We note that the agreement of HCGs 16 and 31 with the cluster fit is likely coincidental because the emission in these systems is largely due to star formation rather than virialization, therefore they should not necessarily agree with the fit to the cluster data.

star formation, superwinds, and accretion) rather than global processes (e.g., virialization). As systems accrete more mass or as the system relaxes, the potential well will deepen and the contribution of individual group members to the diffuse X-ray emission should lessen.

After attempting to subtract the X-ray emission associated with the group members in HCGs, Ponman et al. (1996) found that the $L_{X}-\sigma$ relation was flattened compared to the clusters (i.e., the rate of change in $L_{X}$ as a function of $\sigma$ was slower for groups than for clusters), similar to the dell'Antonio et al. (1994) Einstein study of rich groups, ${ }^{11}$ as well as a subsequent study of CGs by Helsdon \& Ponman (2000). The morphology of the hot gas in CGs may provide an explanation for the groups that disagree with the $L_{X}-T$ and $L_{X}-\sigma$ relations derived from galaxy clusters. As discussed in Section 4.1, the hot IGM that permeates HCG 62 and the X-ray-bright halo centered on HCG 42A have morphologies that are qualitatively similar to the hot gas observed in the cluster environment, i.e., where the gas has been heated by virialization. In the remaining groups, the only diffuse X-ray emission we detect is associated with the individual galaxies rather than the group environment itself. This

\footnotetext{
11 While dell' Antonio et al. (1994) did not subtract the galaxy-linked emission from their data, they did avoid using data in instances where the hot gas was clearly associated with only galaxies and not the group environment.
} 
suggests that the gas is not in hydrostatic equilibrium within the group, and therefore the temperature and luminosity of the total $\mathrm{X}$-ray gas in the system does not trace the group potential as it does in the galaxy clusters.

For completeness, we compare our sample of CGs to studies of the X-ray scaling relations in both normal (i.e., those that are not compact) and fossil groups. With respect to the $L_{X}-T$ relationship, Khosroshahi et al. (2007) and Harrison et al. (2012) noted that fossil groups match well with both clusters and normal groups. Only three of the CGs presented in our study (HCGs 42, 62 , and 92) agree well with the cluster scaling relation within the scatter, though HCG 92 is dominated by emission from a strong shock. The remaining CGs at $T \lesssim 0.65 \mathrm{keV}$ fall below the best fit to the cluster data. In $L_{X}-\sigma$ space, Rines \& Diaferio (2010) showed that normal groups did not deviate from the relation for clusters, while Khosroshahi et al. (2007) found that fossil groups are consistent with the best fit to the cluster data, though more X-ray luminous than the normal groups in their sample. Our sample of CGs falls systematically below the observed $L_{X}-\sigma$ relation for clusters with the exceptions of HCGs 16 and 31 , which are dominated by vigorous star formation. This may be in agreement with the interpretation of merging systems being X-ray underluminous for their velocity dispersions (e.g., Rasmussen et al. 2006; Popesso et al. 2007). We caution the reader that in most cases the velocity dispersions of the groups are measured from very few galaxies, therefore they likely do not accurately represent the three dimensional dispersions that are assumed by the $L_{X}-\sigma$ relation.

Based on the discrepancies between the CGs and the X-ray cluster scaling relations, we postulate that systems similar to the low-mass, $X$-ray faintest groups in our study should not be considered analogs to clusters, with the possible exception of HCG 62, which lies close to the cluster data in both $L_{X}-T$ and $L_{X}-\sigma$ within the scatter. It is important to note that this dissimilarity between the low-mass groups and clusters does not preclude these systems from becoming more cluster-like if they somehow become similar to the more massive, rich groups in our sample (e.g., by accreting additional members and continuing strong interactions to liberate gas into the IGM). However, it is unlikely that rich groups today formed from poor groups like those observed in the current epoch. Therefore the exact mechanism whereby the poor groups in our sample could become more cluster-like remains unknown. We do note, however, that there could be extended, faint emission that is undetectable in the available data (e.g., the X-ray IGM of HCG 16; Belsole et al. 2003). Deeper observations may reveal cooler, lower luminosity gas associated with entire groups rather than the galaxies, which could shed light on the relationship between poor groups and more cluster-like systems.

\subsection{Comparison of the X-Ray Data with H I Gas}

Previous work has shown that relatively $\mathrm{H}$ i rich CGs contain galaxies that exhibit mid-IR colors dominated by star formation with correspondingly high SSFRs measured from UV+24 $\mu \mathrm{m}$ fluxes, while groups deficient in $\mathrm{H}$ i have more quiescent colors and low SSFRs (Johnson et al. 2007; Walker et al. 2010, 2012; Tzanavaris et al. 2010). This suggests an evolutionary sequence of CGs in which the H I gas is processed either through star formation or ionization by the group potential (the velocity dispersions imply virial temperatures of $\sim 0.08-0.3 \mathrm{keV}$; VerdesMontenegro et al. 2001; Johnson et al. 2007). Therefore, we expect that the most $\mathrm{H}$ i abundant groups should have very weak diffuse X-ray emission, while the H i poor groups should have the brightest X-ray luminosities. Furthermore, the morphology of the H I gas may dictate how it is processed, i.e., through star formation or by virialization in the IGM of CGs with neutral gas confined to the galaxies or stripped into the IGM, respectively (cf. Konstantopoulos et al. 2010). Throughout this section, we use the H I abundance type notation from Johnson et al. (2007). Types I, II, and III indicate decreasing $\mathrm{H}$ I abundance relative to the group dynamical mass, respectively. Johnson et al. (2007) quantitatively define these $\mathrm{H}_{\mathrm{I}}$ mass types as (I) $\log \left(M_{\mathrm{HI}}\right)$ / $\log \left(M_{\text {dyn }}\right) \geqslant 0.9$, (II) $0.9>\log \left(\mathrm{M}_{\mathrm{HI}}\right) / \log \left(M_{\text {dyn }}\right) \geqslant 0.8$, and (III) $\log \left(M_{\mathrm{HI}}\right) / \log \left(M_{\text {dyn }}\right)<0.8$. The H I evolutionary types for each group are listed in the last column of Table 3.

We calculate the $\mathrm{H}_{\mathrm{I}}$ to dynamical-mass ratios for the CGs in our sample using the most accurate velocities available from NED, the two galaxy median separators from Hickson et al. (1992), and total group H I masses from Green Bank Telescope, Arecibo Observatory, and Effelsberg $100 \mathrm{~m}$ Antenna single dish measurements by both Verdes-Montenegro et al. (2001) and Borthakur et al. (2010). ${ }^{12}$ Subtypes based on the morphology of the H I gas, which we qualitatively assessed from Very Large Array (VLA) observations, are included as part of the proposed evolutionary sequence of CGs (Konstantopoulos et al. 2010). Type A groups are those in which the neutral gas is confined to the individual group members, while type B groups have H I gas distributed between the galaxies and not centered on any particular member(s). We first test the use of the $\mathrm{H}$ i to dynamical-mass ratio as a descriptor for group evolutionary state by comparing it to the group E/S0 galaxy fraction for the main galaxies. Using the H I mass to dynamical-mass ratio to characterize the evolutionary state is preferred over the $\mathrm{E} / \mathrm{S} 0$ fraction because the mass ratio is a continuous distribution, while the E/S0 fraction values are discrete due to the small number of relatively massive members in each group. To quantify the relationship, we used the ASURV statistical package (Lavalley et al. 1992), which implements the methods presented in Isobe et al. (1986), to compute the Spearman rank correlation coefficient. This test measures how well the data are fit by a single monotonic function. From Figure 5, we find that the H I mass normalized by the group dynamical mass decreases with increasing E/S0 galaxy fraction (67\% probability from Spearman test). This result is expected if the H I to dynamicalmass ratio is indeed a tracer of the evolutionary state of the system.

When we examine how $L_{X}$ changes with $\mathrm{H}_{\mathrm{I}}$ mass ratio, we observe that the H I poor type III HCGs 42, 59, 62, and 90 are $\mathrm{X}$-ray brighter compared to more $\mathrm{H}$ I rich groups as expected if the gas has been processed by star formation or heated by the group potential. Of the type II groups, we note that HCG 92 appears to be nearly as bright as the H I poor CGs, probably due to the shock front created by the high-velocity intruder galaxy in the group (e.g., Trinchieri et al. 2003; O'Sullivan et al. 2009). The only H I rich type I in our study is HCG 31, which contains diffuse X-ray emission linked to star formation activity. Figure 6 shows the X-ray luminosity as a function of the $\mathrm{H}$ I mass ratio. From the figure, we can see that compared to the hot gas in other type III groups, HCGs 59 and 90 are 1-2 orders of magnitude fainter than the remaining H I-poor HCGs. In the case of HCG 90, this could stem from the exclusion of galaxy A from the X-ray analysis due to its bright Seyfert 2 nucleus (see the Appendix); however, it is unlikely that including the diffuse

\footnotetext{
12 The Hi masses of HCGs 16 and 62 are lower and upper limits, respectively. The lower limit of the mass in HCG 16 is due to the large angular size of the $\mathrm{H}$ i envelope, which extends beyond the Green Bank Telescope beam.
} 


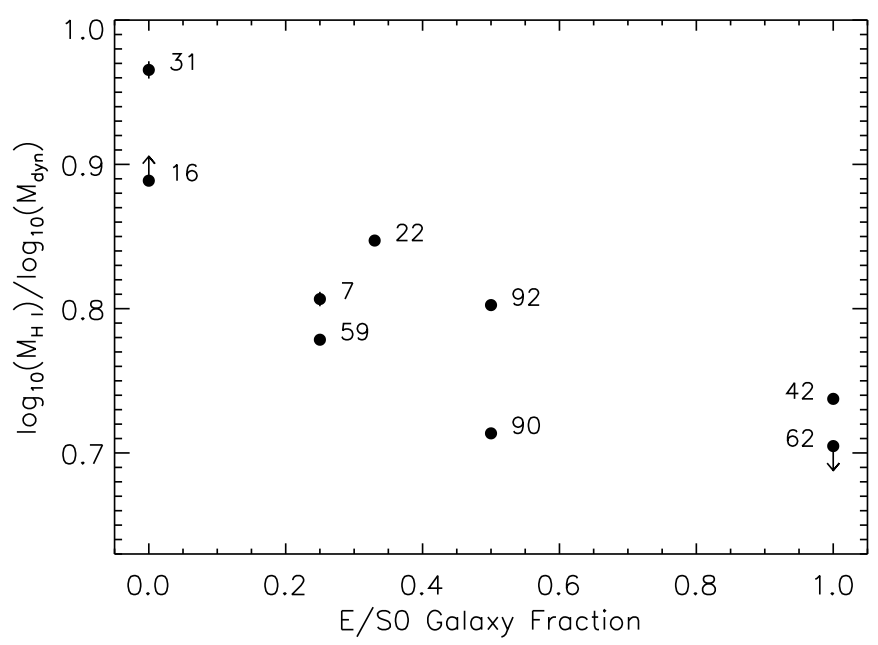

Figure 5. H I gas mass ratio as a function of the main group member morphologies. The Hi mass relative to the group dynamical mass decreases as the fraction of E/S0 galaxies increases. The general trend of the data points indicates that CGs exhaust their $\mathrm{H}_{\mathrm{I}}$ reservoirs (i.e., $M_{\mathrm{H}_{\mathrm{I}}} \lesssim 0.1 \% M_{\mathrm{dyn}}$ ) when approximately $50 \%$ of the main group members have $\mathrm{E} / \mathrm{S} 0$ morphologies.

emission from 90A would increase the total group luminosity by an order of magnitude to bring it to the level of the brighter type III groups.

From Figure 6, we note that if we include the $\mathrm{H}$ I distribution subtypes of the groups, then there may be two distinct trends between the total diffuse X-ray luminosity and the H I evolutionary type. Specifically, the type A groups (H I confined to galaxies) appear to be consistently less luminous in X-rays compared to type B groups (H I in the IGM). Based on this tentative result, it is reasonable to predict that HCG 62 should have any remaining $\mathrm{H}$ I dispersed throughout its IGM, while H I gas in HCG 59 should be mostly contained in and around its group members. However, we note that this result is preliminary and that more data are required to quantitatively assess the likelihood that the two H I gas morphologies are distinct populations in X-ray luminosity. If we assume these $\mathrm{H}$ I morphology classifications for HCGs 59 and 62 to increase the number of data points in each sample to the minimum required, a two sample K-S test gives a $95 \%$ probability that the $\mathrm{H}$ I subtypes are two distinct populations in X-ray luminosity. To examine how our assumption concerning the H I subtypes of HCGs 59 and 62 influenced the test, we perform a second $\mathrm{K}-\mathrm{S}$ test in which we switch the $\mathrm{HI}$ morphology classifications for these groups (i.e., HCG 59 has intragroup $\mathrm{H}$ I, while 62 has $\mathrm{H}$ I only in the galaxies). This results in an $12 \%$ probability that the two H I morphologies represent distinguishable populations in $L_{X}$. Due to the small sample size, we cannot determine if this distinction is statistically significant, and further data are required to clarify this phenomenon.

We note that the mix of galaxy morphologies in the groups complicates the comparison of the $\mathrm{H}$ I and X-ray gas. However, Verdes-Montenegro et al. (2001) and Borthakur et al. (2010) reported on the observed Hi masses in these groups and the predicted $\mathrm{H}$ I masses based on the group member luminosities and morphologies using relations from Haynes \& Giovanelli (1984) who examined H I in a sample of isolated galaxies. From this, we can compute $\mathrm{H}$ I deficiencies for the groups in our sample, where deficiency describes the ratio of the Hi mass observed to that predicted (in contrast to our use of $\mathrm{H}$ I rich and poor, which describes the relative neutral gas mass normalized to the dynamical mass). Using the nomenclature of Borthakur et al. (2010; i.e., "heavily" deficient, "slightly" deficient, and

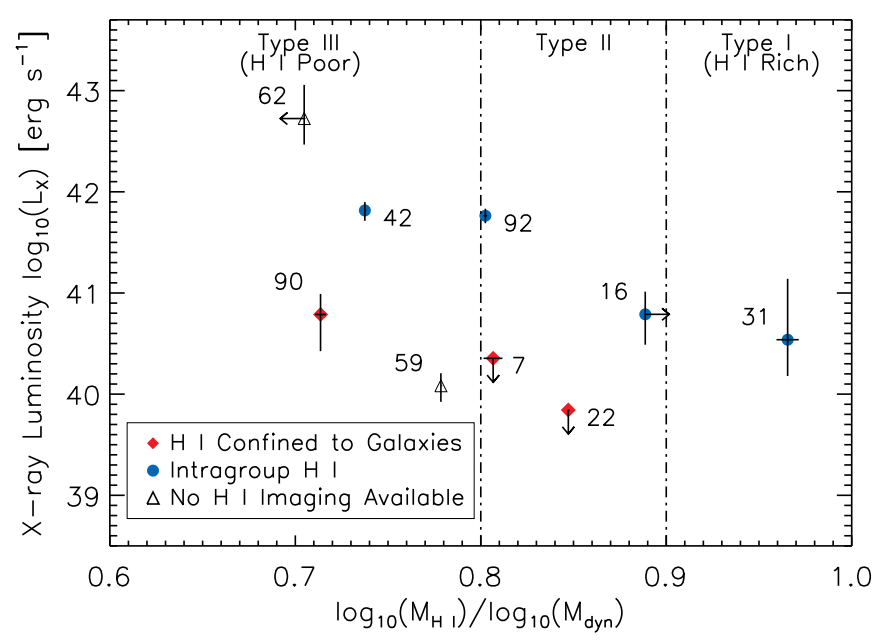

Figure 6. Distribution of diffuse X-ray luminosity in CGs as a function of the ratio of $\mathrm{HI}$ mass to dynamical mass used in the evolutionary typing scheme defined by Johnson et al. (2007). Neutral gas masses are taken from the Green Bank Telescope, Arecibo Observatory, and $100 \mathrm{~m}$ Effelsberg Antenna single dish measurements by Verdes-Montenegro et al. (2001) and Borthakur et al. (2010), while the $\mathrm{H}$ I morphologies are qualitatively assessed from VLA interferometric observations, which we lack for HCGs 59 and 62 . Note that the values of the H I mass in HCGs 62 and 16 are upper and lower limits, respectively, and the plotted H I mass ratio corresponds to these limits. Furthermore, uncertainties in the Hi masses from Verdes-Montenegro et al. (2001) are not available, therefore we only plot error bars in the H I mass ratio for data from Borthakur et al. (2010). After separating the groups by H I distribution subtype based on Konstantopoulos et al. (2010), there appears to be a distinction in the X-ray luminosity between the two populations. Due to the small number of groups in our sample, more data are required to concretely determine if this difference is real.

(A color version of this figure is available in the online journal.)

normal groups contain $<1 / 3$, between $1 / 3$ and $2 / 3$, and $>2 / 3$ of their predicted $\mathrm{H}_{\mathrm{I}}$, respectively), we find that the $\mathrm{X}$-ray fainter groups are either heavily deficient $(7,22$, and 90) or normal (59) groups, while the brightest groups are either slightly deficient $\left(16,31,62,{ }^{13}\right.$ and 92 or normal (42) groups.

Because the gas is not in hydrostatic equilibrium (as indicated by the diffuse X-ray morphology; see Section 4.1), and in many cases linked to the individual group members rather than the environment, we cannot use the temperature to calculate the group hot gas mass (such a procedure assumes hydrostatic equilibrium in three dimensions). However, if we did assume that the gas is in hydrostatic equilibrium, then the narrow range of temperatures implies a similarly narrow range of masses (Fabricant et al. 1980). The X-ray contours in Figure 1 make this unconvincing due to the extremely large number densities and/or metal abundances necessary to make the hot gas masses of groups such as HCG 16 similar to ones such as HCGs 90 or 62. If we instead hypothesize that the X-ray gas mass is proportional to the bolometric X-ray luminosity, i.e., that there exists a $L_{X}-M_{X}$ relation for groups (as in Zhang et al. 2011 for clusters), then we can compare the relative X-ray luminosities of the groups as a proxy for their relative hot gas masses. That the X-ray fainter groups for a given $\mathrm{H}$ I mass ratio are deficient in $\mathrm{H} \mathrm{I}$ and that all of the CGs have similar X-ray temperatures suggest two scenarios: either (1) additional X-ray gas has too low surface brightness to detect in the available data or (2) some fraction of it is missing from the groups. If the Hi was not

\footnotetext{
13 This group is likely heavily deficient as its $\mathrm{H}$ I mass is actually an upper limit and the ratio of the limit to the predicted value is $35 \%$ (Verdes-Montenegro et al. 2001).
} 


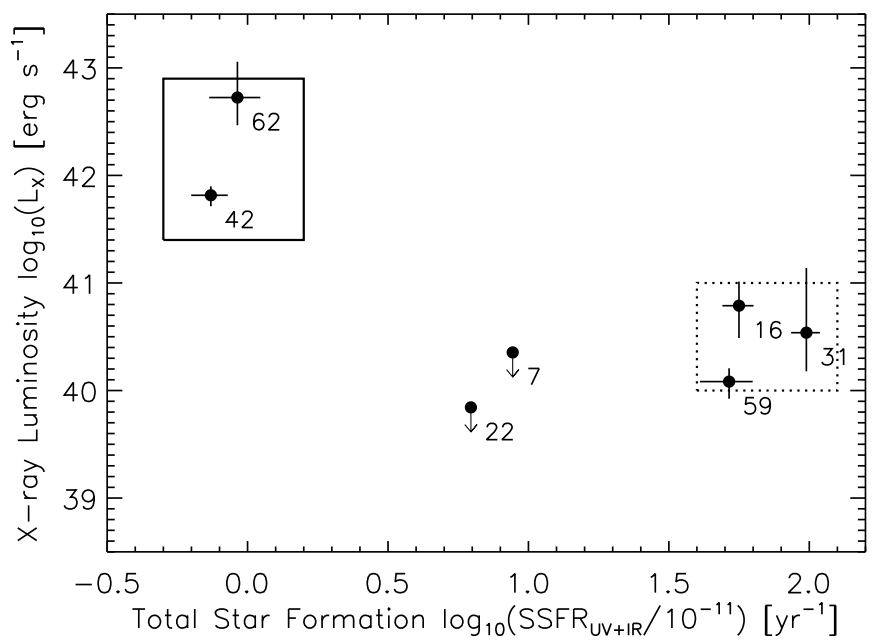

Figure 7. X-ray luminosity as a function of the specific star formation rate for the seven groups with SSFRs from Tzanavaris et al. (2010). The groups primarily fall into two classes: quiescent, X-ray brighter systems (solid box) and starforming, X-ray fainter systems (dotted box). The boxes are only used to identify the groups in these two regimes, therefore the absolute positions and sizes of the boxes in the figure do not necessarily carry physical meaning. At the low and high SSFRs, the processes that give rise to the X-ray emission are dominated by the group potential and local means (e.g., superwinds), respectively.

converted to hot gas, then where is the missing gas in CGs? The least massive groups in our sample have velocity dispersions that correspond to virial temperatures of only $\sim 0.08 \mathrm{keV}$; however, if the gas is cooler than the virial theorem implies, then it may be in the form of a diffuse UV intragroup medium. Further data are required to fully explore this phenomenon.

\subsection{Diffuse X-Ray Emission and Specific Star Formation Rates}

We also compare the X-ray emission of seven groups in our sample to the total group SSFRs for the main group members calculated from the UV+24 $\mu \mathrm{m}$ fluxes measured by Tzanavaris et al. (2010) with corrections from P. Tzanavaris (2012, private communication) in Figure 7, and find that there is a distinction in $L_{X}$ for detected groups on either side of the SSFR gap (data are not available for HCGs 90 and 92). We exclude dwarf group members with measured SSFRs because HCG 31 is the only $\mathrm{CG}$ in our sample that has such measurements of its dwarf population, which increases the total group SSFR by several orders of magnitude due to the combination of relatively low to moderate SFRs and small stellar masses in star-forming dwarf galaxies. In our sample, CGs containing star-forming galaxies all share similar X-ray luminosities and are 1-2 orders of magnitude fainter than groups with low total SSFRs. This may indicate an "X-ray gap" analogous to the SSFR and mid-IR color gaps found by Tzanavaris et al. (2010) and Walker et al. (2010, 2012). We note that HCGs 7 and 22 have SSFRs that lie within the gap range of $(0.3-1.8) \times 10^{-11} \mathrm{yr}^{-1}$, and neither has any detected diffuse X-ray emission.

The brighter $L_{X}$ values associated with the low SSFR groups are due to the advanced evolutionary stage of these environments. HCGs 42 and 62 have very low Hi to dynamical-mass ratios, while simultaneously their relatively massive galaxies are entirely E/S0 types. From the evolutionary scenario presented by Verdes-Montenegro et al. (2001), and the fact that compact environments are favorable to the tidal stripping of gas from galaxies (Freeland et al. 2009), we should expect that these older CGs have removed the cool gas from the galaxies and heated it in the intragroup medium. Conversely, the star-forming groups HCGs 16, 31, and 59 have relatively faint $\mathrm{X}$-ray emission associated with them. These groups also all exhibit only galaxy-linked emission, as expected from their relatively young evolutionary states. Finally, neither of the intermediate SSFR groups, HCGs 7 and 22, are detected by Chandra. From the figure, we find that there are two possible evolutionary scenarios with respect to the total group SSFR: (1) the groups move from the lower right portion of Figure 7 to the upper left, i.e., star-forming and X-ray fainter to quiescent and X-ray brighter, though perhaps not monotonically; or (2) there exists at least one more evolutionary track in which star formation in CG galaxies declines while gas is not stripped from the disks and/or heated. In particular, studies of groups similar to HCGs 7 and 22 may provide further insight.

\section{SUMMARY}

We detect diffuse X-ray emission in seven of nine of the CGs in our sample with temperatures ranging from $0.6-0.72 \mathrm{keV}$ and bolometric X-ray luminosities between $10^{40.4}$ and $10^{42.2} \mathrm{erg} \mathrm{s}^{-1}$. The groups exhibit a wide range of velocity dispersions $\left(56-343 \mathrm{~km} \mathrm{~s}^{-1}\right), \log _{10}\left(M_{\mathrm{H} \mathrm{I}}\right) / \log _{10}\left(M_{\mathrm{dyn}}\right)(0.70-0.97)$, and morphological fractions from spiral-only groups to systems rich with E/S0 galaxies.

Based on the hot gas morphologies, we find that the X-ray emission likely arises due to both local processes (i.e., star formation, nuclear activity, and tidal interactions) and global processes (i.e., heating by the group potential). In dynamically unevolved (i.e., low-mass, low-velocity dispersion) systems, the observable diffuse X-ray emission is dominated by local processes. The X-ray brighter groups (for a given $\mathrm{H}$ I mass ratio) have emission that stems from both an extended diffuse component (i.e., a true intragroup medium) and galaxy-linked emission, while emission detected in the X-ray fainter groups is only associated with the individual galaxies.

HCGs 31, 42, 59, and 92 have X-ray luminosities in agreement with the predicted values from the $L_{X}-T$ scaling relation from clusters, though the error in the temperatures of HCGs 31 and 59 are large. Furthermore, HCGs 16 and 31 agree with the $L_{X}-\sigma$ relation. In both $L_{X}-T$ and $L_{X}-\sigma$, HCG 62 appears to lie within the scatter of the cluster data and is similar to fainter $L_{X}$ clusters. The agreement between HCGs 16 and 31 with the $L_{X}-\sigma$ relation are likely coincidental because the scaling relation is predicted from the virial theorem (i.e., $L_{X} \propto \sigma^{4}$ ), and the X-ray emission from these two systems is clearly dominated by star formation rather than virialization. When the groups disagree with the cluster scaling relations, particularly in $L_{X}-\sigma$ space, this indicates that the groups are not simply scaled-down analogs to galaxy clusters. Furthermore, given that the hot gas in the low-mass (i.e., low-velocity dispersion) systems is found to be isolated to the group members rather than throughout the intragroup medium, we conclude that galaxy clusters are not a proper comparison class of objects for these groups (noting the possible exception of HCG 62).

We also find that there may be a relationship between $L_{X}$ and how the $\mathrm{H}$ I gas is distributed: preliminary evidence suggests that CGs with gas stripped from the galaxies are brighter in X-rays than groups with $\mathrm{H}$ I confined to the members, possibly due to strong multi-galaxy interactions that dispersed neutral gas into the intragroup medium and triggered star formation. However, the X-ray faintest groups are also more heavily deficient in $\mathrm{H}$ I, implying that there may be some fraction of missing gas, possibly too cool to emit in X-rays or with too low surface brightness to detect. Finally, we note that groups dominated by 
local heating mechanisms have high UV+24 $\mu \mathrm{m}$ SSFRs, while groups with gas heated by the group potential have low SSFRs. The values of $L_{X}$ between these two categories span $\sim 2$ orders of magnitude and may indicate the presence of an "X-ray gap" in CGs similar to the SSFR and mid-IR color gaps found by Tzanavaris et al. (2010) and Walker et al. (2010, 2012).

The faintest $L_{X}$ groups appear to be at very early stages in their evolution, perhaps coming together for the first time as is indicated by their low fractions of E/S0 galaxies. The influence of multi-galaxy interactions on liberating neutral gas from the galaxies and depositing it into the intragroup medium early in the group lifetime (e.g., as seen in HCG 16) appears to have an effect on the ability of these groups to evolve into more cluster-like systems with respect to the hot gas distribution (e.g., HCG 62).

\subsection{Future Work}

Expanding the sample with appropriate observations of groups from the Chandra and XMM-Newton archives is the logical next step. This will give us a larger sample with which to study the relation between the hot gas and the evolution of group environment (e.g., how the X-ray luminosity varies with $\mathrm{H}_{\mathrm{I}}$ mass ratio). Furthermore, continuing to examine the differences between compact, loose, and fossil groups will demonstrate how efficient the compact environment processes gas. Inclusion of multiwavelength data will help to facilitate comparison of the hot gas to gas in cooler states (e.g., cold molecular). HCG 16 in particular is an interesting group worth more study; the presence of two potential M82-like superwinds in a single system presents an interesting case study for tidally induced star formation and how superwinds drive hot gas into the intragroup medium.

T.D.D. and S.C.G. thank the Natural Science and Engineering Research Council of Canada and the Ontario Early Researcher Award Program for support. This work was partially supported by the ACIS Instrument Team contract SAO SV4-74018 (PI: G. Garmire). W.N.B. thanks NASA ADP grant NNX10AC99G and NSF grant AST-1108604 for support. The authors are grateful to the anonymous referee for providing thoughtful feedback that improved the manuscript. T.D.D. also thanks Tesla Jeltema, Allison R. Hill, and Alexander DeSouza for their helpful comments. The Institute for Gravitation and the Cosmos is supported by the Eberly College of Science and the Office of the Senior Vice President for Research at the Pennsylvania State University. J.C.C., I.S.K., and C.G. acknowledge funding that was provided through Chandra Award No. GO8-9124B issued by the Chandra X-Ray Observatory Center, which is operated by the Smithsonian Astrophysical Observatory under NASA contract NAS8-03060, and by the National Science Foundation under award 0908984. This research has made use of the NASA/IPAC Extragalactic Database (NED) which is operated by the Jet Propulsion Laboratory, California Institute of Technology, under contract with the National Aeronautics and Space Administration.

\section{APPENDIX}

\section{NOTES ON INDIVIDUAL GROUPS}

In addition to the unique aspects of the X-ray analysis and properties of the diffuse emission, we also list the center R.A. and decl., shape, and dimensions of the extraction region for each group.
$H C G$ 7. Diffuse emission in this group was not detected above the background in the Chandra data. The circular extraction region with radius 3.9 was centered at R.A. $=00^{\mathrm{h}} 39^{\mathrm{m}} 23^{\mathrm{s}} .9$ and decl. $=+00^{\circ} 52^{\prime} 15^{\prime \prime} .4$.

$H C G$ 16. The shallow observation of this group prevented detection of a true intragroup medium (i.e., the $L_{X}=2.4 \times$ $10^{40} \mathrm{erg} \mathrm{s}^{-1}$ IGM, corrected to our cosmology, found by Belsole et al. 2003 with $45 \mathrm{ks}$ of XMM data); however, hot gas associated with the individual galaxy members was detected. The extracted spectrum corresponds to the area surrounding galaxies A, B, C, and $\mathrm{D}$, but does not include galaxy $\mathrm{X}$, which is far removed from the group center and located far from the S3 aimpoint of the observation. The rectangular 7!.2 $\times 3.7$ extraction region was centered at R.A. $=02^{\mathrm{h}} 09^{\mathrm{m}} 33.0$ and decl. $=-10^{\circ} 09^{\prime} 05^{\prime \prime} .8$ with P.A. $=36^{\circ}$.

$H C G$ 22. Diffuse emission in this group was not detected above the background in the Chandra data. The rectangular $6.5 \times 6^{\prime} .9$ extraction region was centered at R.A. $=03^{\mathrm{h}} 03^{\mathrm{m}} 30^{\mathrm{s}} .1$ and decl. $=-15^{\circ} 39^{\prime} 27^{\prime \prime} .3$ with P.A. $=22^{\circ}$. We note that this region includes a background pair of galaxies to the southeast of the main group members; however, any emission from this pair is negligible and does not adversely affect the upper limit on the X-ray luminosity of HCG 22.

$H C G$ 31. The $\mathrm{S} / \mathrm{N}$ of the detection is only marginally above the threshold required for a detection $(\mathrm{S} / \mathrm{N}=3)$, and therefore the properties of the hot gas in this system are poorly constrained. However, this is the first detection of diffuse X-ray emission in this group. The extraction region covers the massive group members and the southern tidal tail where the majority of star formation is occurring within the group (Gallagher et al. 2010). Based on the reservoir of H I gas in the group, Gallagher et al. (2010) predicted that conversion of $75 \%$ of the neutral gas into stellar mass over a 150 Myr episode of star formation would generate $\sim 2 \times 10^{39} \mathrm{erg} \mathrm{s}^{-1}$ of X-ray emission. However, this is an order of magnitude below the observed value of $L_{X}$ from the Chandra data. The elliptical $2 ! 1 \times 1$. 3 extraction region was centered at R.A. $=05^{\mathrm{h}} 01^{\mathrm{m}} 39^{\mathrm{s}} .7$ and decl. $=-04^{\circ} 16^{\prime} 15^{\prime \prime} .8$ with P.A. $=44^{\circ}$

$H C G 42$. We extracted spectra from both the region around galaxy A, where the diffuse X-ray emission is most readily apparent, and from a region containing all of the massive group members. However, the count rates obtained from both extraction regions were consistent within errors. Therefore, we find that the majority of the X-ray emission in the system is associated with galaxy A. To ensure that we do not exclude any extended emission, we compare our value with that derived from ROSAT PSPC data using the extended radius of the X-ray emission from Mulchaey \& Zabludoff (1998). This radius corresponds to the distance at which the X-ray emission falls to $20 \%$ of its peak value; in $\mathrm{HCG} 42$, this radius is $8^{\prime}$. Comparison with the flux extracted from the ROSAT data shows that our value of the flux within our extraction region matches that obtained with an $8^{\prime}$ radius. The circular extraction region with radius 3.4 was centered at R.A. $=10^{\mathrm{h}} 00^{\mathrm{m}} 21^{\mathrm{s}} .0$ and decl. $=$ $-19^{\circ} 38^{\prime} 48^{\prime \prime} .5$.

HCG 59. As for HCG 31, the X-ray emission from this group is extremely weak; however, the $\mathrm{S} / \mathrm{N}$ is sufficient to classify this group as a detection. Due to the low $\mathrm{S} / \mathrm{N}$, the values derived from model fits to the extracted spectra are poorly constrained. Though it is unlikely that the value of the temperature could span two orders of magnitude (as indicated by the upper $90 \%$ confidence error estimate), we include this error in subsequent figures for consistency. The $3.9 \times 2^{\prime}$ elliptical extraction region 
was centered at R.A. $=11^{\mathrm{h}} 48^{\mathrm{m}} 26^{\mathrm{S}} .5$ and decl. $=12^{\circ} 43^{\prime} 10^{\prime \prime} \cdot 2$ with P.A. $=0.3$.

$H C G$ 62. Previous work has found that there are cavities in the X-ray emission around HCG 62 (the result of active galactic nucleus jets and lobes due to the Seyfert 2 nucleus in galaxy A) that lack high-frequency radio emission, but do have powerful low-frequency emission (Dong et al. 2010; Gitti et al. 2010; Giacintucci et al. 2011; O'Sullivan et al. 2011). The extraction region does not include galaxy $\mathrm{D}$, which is far to the south with respect to the other group members, and has negligible X-ray emission. During model fitting, we found that a model with a single MEKAL component was insufficient to properly fit the observed spectrum, therefore we model this group with two separate plasmas. We note that Mulchaey \& Zabludoff (1998) found that the diffuse emission extends much farther from the group center than is evident in the Chandra data, and well beyond the field of view of the ACIS CCDs. Using the extended X-ray emission radius of 24 '.2 from Mulchaey \& Zabludoff (1998), we found that our measured absorbed flux was a factor of 3.1 lower than that found from the ROSAT PSPC data and thus correct our measurements by this amount. Note that we apply this correction to both components of the emission in addition to the total luminosity, therefore the luminosities of the hot and cold component should be considered upper limits. The circular extraction region with radius 1.5 was centered at R.A. $=12^{\mathrm{h}} 53^{\mathrm{m}} 06^{\mathrm{S}} .0$ and decl. $=-09^{\circ} 12^{\prime} 11^{\prime \prime} .6$.

$H C G$ 90. The spectral extraction region for this group is centered on galaxies B, C, and D that are currently interacting with one another and are embedded within a halo of diffuse optical light (White et al. 2003). The region excludes the brightest member (90A), which is located $\sim 6.9$ (68 kpc) from the other three massive, interacting group members and contains a powerful Seyfert 2 nucleus. This bright AGN complicates analysis of the diffuse emission with bright readout streaks and substantial pileup on the I3 CCD. The 5'.3 $\times 4^{\prime} .1$ elliptical extraction region was centered at $\mathrm{RA}=22^{\mathrm{h}} 02^{\mathrm{m}} 04^{\mathrm{s}} .5$ and decl. $=$ $-31^{\circ} 58^{\prime} 51^{\prime \prime} .9$ with P.A. $=100^{\circ}$.

$H C G$ 92. The known primary source of heating for the hot gas is a shock front caused by the high-velocity intruder galaxy NGC $7318 \mathrm{~B}$ as it moves at $\sim 850 \mathrm{~km} \mathrm{~s}^{-1}$ through the intragroup medium (Pietsch et al. 1997). Numerous interactions have occurred in the group in the past $\sim 500$ Myr leading to tidal tails and debris (e.g., Fedotov et al. 2011; Hwang et al. 2012). These frequent interactions in the group likely caused gas to be stripped from the member galaxies and be deposited into the intragroup medium (Moles et al. 1997; Guillard et al. 2012). We note that the presence of the shock in the intragroup medium could have non-thermal X-ray emission that is not included in the MEKAL model fit. The circular extraction region with radius 2.5 was centered at R.A. $=22^{\mathrm{h}} 35^{\mathrm{m}} 59^{\mathrm{s}} .5$ and decl. $=33^{\circ} 58^{\prime} 03^{\prime \prime} .2$.

\section{REFERENCES}

Akritas, M. G., \& Bershady, M. A. 1996, ApJ, 470, 706

Anders, E., \& Grevesse, N. 1989, GeCoA, 53, 197

Arnaud, K. A. 1996, in ASP Conf. Ser. 101, Astronomical Data Analysis Software and Systems V, ed. G. H. Jacoby \& J. Barnes (San Francisco, CA: ASP), 17

Arnaud, M., \& Raymond, J. 1992, ApJ, 398, 394

Arnaud, M., \& Rothenflug, R. 1985, A\&AS, 60, 425

Belsole, E., Sauvageot, J.-L., Ponman, T. J., \& Bourdin, H. 2003, A\&A, 398, 1

Borthakur, S., Yun, M. S., \& Verdes-Montenegro, L. 2010, ApJ, 710, 385

Broos, P. S., Townsley, L. K., Feigelson, E. D., et al. 2010, ApJ, 714, 1582

Chartas, G., Gupta, V., Garmire, G., et al. 2002, ApJ, 565, 96

da Costa, L. N., Willmer, C. N. A., Pellegrini, P. S., et al. 1998, AJ, 116, 1 de Carvalho, R. R., Ribeiro, A. L. B., Capelato, H. V., \& Zepf, S. E. 1997, ApJS, 110,1

dell'Antonio, I. P., Geller, M. J., \& Fabricant, D. G. 1994, AJ, 107, 427

de Vaucouleurs, G., de Vaucouleurs, A., Corwin, H. G., Jr., et al. 1991, Third Reference Catalogue of Bright Galaxies. Volume I: Explanations and References. Volume II: Data for Galaxies between $0^{h}$ and $12^{h}$. Volume III: Data for Galaxies between $12^{h}$ and $24^{h}$ (New York: Springer)

Diaferio, A., Geller, M. J., \& Ramella, M. 1994, AJ, 107, 868

Dong, R., Rasmussen, J., \& Mulchaey, J. S. 2010, ApJ, 712, 883

Fabricant, D., Lecar, M., \& Gorenstein, P. 1980, ApJ, 241, 552

Falco, E. E., Kurtz, M. J., Geller, M. J., et al. 1999, PASP, 111, 438

Fedotov, K., Gallagher, S. C., Konstantopoulos, I. S., et al. 2011, AJ, 142, 42

Fixsen, D. J., Cheng, E. S., Gales, J. M., et al. 1996, ApJ, 473, 576

Freeland, E., Stilp, A., \& Wilcots, E. 2009, AJ, 138, 295

Freeman, P. E., Kashyap, V., Rosner, R., \& Lamb, D. Q. 2002, ApJS, 138, 185 Gallagher, S. C., Charlton, J. C., Hunsberger, S. D., Zaritsky, D., \& Whitmore, B. C. 2001, AJ, 122, 163

Gallagher, S. C., Durrell, P. R., Elmegreen, D. M., et al. 2010, AJ, 139, 545

Giacintucci, S., O’Sullivan, E., Vrtilek, J., et al. 2011, ApJ, 732, 95

Gitti, M., O’Sullivan, E., Giacintucci, S., et al. 2010, ApJ, 714, 758

Gottesman, S. T., \& Weliachew, L. 1977, ApJ, 211, 47

Guillard, P., Boulanger, F., Pineau des Forêts, G., et al. 2012, ApJ, 749, 158

Harrison, C. D., Miller, C. J., Richards, J. W., et al. 2012, ApJ, 752, 12

Haynes, M. P., \& Giovanelli, R. 1984, AJ, 89, 758

Helsdon, S. F., \& Ponman, T. J. 2000, MNRAS, 315, 356

Helsdon, S. F., Ponman, T. J., O’Sullivan, E., \& Forbes, D. A. 2001, MNRAS, 325,693

Hickox, R. C., \& Markevitch, M. 2006, ApJ, 645, 95

Hickson, P. 1982, ApJ, 255, 382

Hickson, P., Mendes de Oliveira, C., Huchra, J. P., \& Palumbo, G. G. 1992, ApJ, 399,353

Huchra, J., Latham, D. W., da Costa, L. N., Pellegrini, P. S., \& Willmer, C. N. A. $1993, \mathrm{AJ}, 105,1637$

Huchtmeier, W. K. 1994, A\&A, 286, 389

Huchtmeier, W. K. 1997, A\&A, 325, 473

Hwang, J.-S., Struck, C., Renaud, F., \& Appleton, P. N. 2012, MNRAS, 419, 1780

Isobe, T., Feigelson, E. D., \& Nelson, P. I. 1986, ApJ, 306, 490

Jeltema, T. E., Binder, B., \& Mulchaey, J. S. 2008, ApJ, 679, 1162

Johnson, K. E., Hibbard, J. E., Gallagher, S. C., et al. 2007, AJ, 134, 1522

Jones, D. H., Read, M. A., Saunders, W., et al. 2009, MNRAS, 399, 683

Jones, L. R., Ponman, T. J., Horton, A., et al. 2003, MNRAS, 343, 627

Kaastra, J. S. 1992, An X-Ray Spectral Code for Optically Thin Plasmas, Internal SRON-Leiden Report dd. 1992-12-03

Kaastra, J. S. 1993, Legacy, 3, 16

Kaastra, J. S., \& Liedahl, D. A. 1995, Legacy, 6, 16

Kalberla, P. M. W., Burton, W. B., Hartmann, D., et al. 2005, A\&A, 440, 775

Karachentsev, I. D. 2005, AJ, 129, 178

Kereš, D., Katz, N., Weinberg, D. H., \& Davé, R. 2005, MNRAS, 363, 2

Khosroshahi, H. G., Ponman, T. J., \& Jones, L. R. 2007, MNRAS, 377, 595

Konstantopoulos, I. S., Bastian, N., Smith, L. J., et al. 2009, ApJ, 701, 1015

Konstantopoulos, I. S., Gallagher, S. C., Fedotov, K., et al. 2010, ApJ, 723, 197

Konstantopoulos, I. S., Gallagher, S. C., Fedotov, K., et al. 2012, ApJ, 745, 30

Lavalley, M. P., Isobe, T., \& Feigelson, E. D. 1992, BAAS, 24, 839

Liedahl, D. A., Osterheld, A. L., \& Goldstein, W. H. 1995, ApJL, 438, 115

Lodders, K. 2003, ApJ, 591, 1220

Mamon, G. A. 1986, ApJ, 307, 426

Mendel, J. T., Ellison, S. L., Simard, L., Patton, D. R., \& McConnachie, A. W. 2011, MNRAS, 418, 1409

Mendes de Oliveira, C., Coelho, P., González, J. J., \& Barbuy, B. 2005, AJ, 130,55

Mendes de Oliveira, C., Plana, H., Amram, P., Bolte, M., \& Boulesteix, J. 1998, ApJ, 507, 691

Mendes de Oliveira, C. L., Temporin, S., Cypriano, E. S., et al. 2006, AJ, 132,570

Meurer, G. R., Hanish, D. J., Ferguson, H. C., et al. 2006, ApJS, 165, 307

Mewe, R., Gronenschild, E. H. B. M., \& van den Oord, G. H. J. 1985, A\&AS, 62, 197

Mewe, R., Lemen, J. R., \& van den Oord, G. H. J. 1986, A\&AS, 65, 511

Moles, M., Sulentic, J. W., \& Marquez, I. 1997, ApJL, 485, 69

Monnier Ragaigne, D., van Driel, W., Schneider, S. E., Balkowski, C., \& Jarrett, T. H. 2003, A\&A, 408, 465

Mulchaey, J. S. 2000, ARA\&A, 38, 289

Mulchaey, J. S., Davis, D. S., Mushotzky, R. F., \& Burstein, D. 2003, ApJS, 145,39

Mulchaey, J. S., \& Zabludoff, A. I. 1998, ApJ, 496, 73 
Nishiura, S., Shimada, M., Ohyama, Y., Murayama, T., \& Taniguchi, Y. 2000, AJ 120,1691

Osmond, J. P. F., \& Ponman, T. J. 2004, MNRAS, 350, 1511

O’Sullivan, E., Giacintucci, S., David, L. P., et al. 2011, ApJ, 735, 11

O'Sullivan, E., Giacintucci, S., Vrtilek, J. M., Raychaudhury, S., \& David, L. P. 2009, ApJ, 701, 1560

Paturel, G., Theureau, G., Bottinelli, L., et al. 2003, A\&A, 412, 57

Pietsch, W., Trinchieri, G., Arp, H., \& Sulentic, J. W. 1997, A\&A, 322, 89

Ponman, T. J., Bourner, P. D. J., Ebeling, H., \& Böhringer, H. 1996, MNRAS, 283,690

Ponman, T. J., Cannon, D. B., \& Navarro, J. F. 1999, Natur, 397, 135

Popesso, P., Biviano, A., Böhringer, H., \& Romaniello, M. 2007, A\&A, 461,397

Rasmussen, J., \& Ponman, T. 2009, in ASP Conf. Ser. 419, Galaxy Evolution: Emerging Insights and Future Challenges, ed. S. Jogee et al. (San Francisco, CA: ASP), 415

Rasmussen, J., Ponman, T. J., Mulchaey, J. S., Miles, T. A., \& Raychaudhury, S. 2006, MNRAS, 373, 653

Rasmussen, J., Ponman, T. J., Verdes-Montenegro, L., Yun, M. S., \& Borthakur, S. 2008, MNRAS, 388, 1245

Ribeiro, A. L. B., de Carvalho, R. R., Coziol, R., Capelato, H. V., \& Zepf, S. E. 1996, ApJ, 463, L5

Rich, J. A., Dopita, M. A., Kewley, L. J., \& Rupke, D. S. N. 2010, ApJ, 721,505
Rines, K., \& Diaferio, A. 2010, AJ, 139, 580

Small, T. A., Ma, C.-P., Sargent, W. L. W., \& Hamilton, D. 1999, ApJ, 524, 31

Sun, M., Voit, G. M., Donahue, M., et al. 2009, ApJ, 693, 1142

Tamburri, S., Trinchieri, G., Wolter, A., et al. 2012, A\&A, 541, A28

Theureau, G., Bottinelli, L., Coudreau-Durand, N., et al. 1998, A\&AS, 130, 333

Tovmassian, H., Plionis, M., \& Torres-Papaqui, J. P. 2006, A\&A, 456, 839

Trinchieri, G., Sulentic, J., Breitschwerdt, D., \& Pietsch, W. 2003, A\&A, 401, 173

Tully, R. B. 1987, ApJ, 321, 280

Tzanavaris, P., Hornschemeier, A. E., Gallagher, S. C., et al. 2010, ApJ, 716,556

Verdes-Montenegro, L., Del Olmo, A., Yun, M. S., \& Perea, J. 2005, A\&A, 430,443

Verdes-Montenegro, L., Yun, M. S., Williams, B. A., et al. 2001, A\&A, 377,812

Walker, L. M., Johnson, K. E., Gallagher, S. C., et al. 2010, AJ, 140, 1254

Walker, L. M., Johnson, K. E., Gallagher, S. C., et al. 2012, AJ, 143, 69

White, P. M., Bothun, G., Guerrero, M. A., West, M. J., \& Barkhouse, W. A. 2003, ApJ, 585, 739

Wilms, J., Allen, A., \& McCray, R. 2000, ApJ, 542, 914

Wu, X.-P., Xue, Y.-J., \& Fang, L.-Z. 1999, ApJ, 524, 22

Zabludoff, A. I., \& Mulchaey, J. S. 1998, ApJ, 496, 39

Zabludoff, A. I., \& Mulchaey, J. S. 2000, ApJ, 539, 136

Zhang, Y.-Y., Andernach, H., Caretta, C. A., et al. 2011, A\&A, 526, A105 